\title{
The CARMA-NRO Orion Survey
}

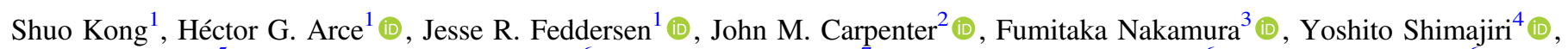

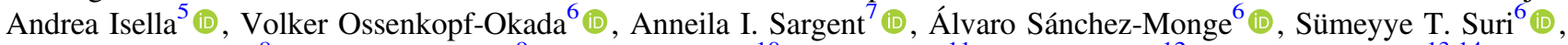

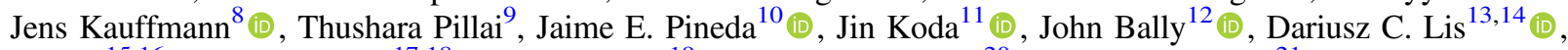
Paolo Padoan ${ }^{15,16}$ (10), Ralf Klessen ${ }^{17,18}$ (1), Steve Mairs ${ }^{19}$ (1) Alyssa Goodman $^{20}$ (1), Paul Goldsmith ${ }^{21}$ (10), Peregrine McGehee ${ }^{22}$, Peter Schilke ${ }^{6}$ (1), Peter J. Teuben ${ }^{23}$ (1), María José Maureira ${ }^{1}$, Chihomi Hara ${ }^{24}$, Adam Ginsburg ${ }^{25}$ (1), Blakesley Burkhart ${ }^{26}$, Rowan J. Smith ${ }^{27}$ (1), Anika Schmiedeke ${ }^{6,10}$, Jorge L. Pineda ${ }^{21}$ (1), Shun Ishii ${ }^{2}$, Kazushige Sasaki ${ }^{28}$, Ryohei Kawabe ${ }^{3}$ (1), Yumiko Urasawa ${ }^{28}$, Shuri Oyamada ${ }^{29}$, and Yoshihiro Tanabe ${ }^{30}$

${ }^{1}$ Department of Astronomy, Yale University, New Haven, CT 06511, USA

2 Joint ALMA Observatory, Alonso de Córdova 3107 Vitacura, Santiago, Chile

${ }^{3}$ National Astronomical Observatory of Japan, 2-21-1 Osawa, Mitaka, Tokyo 181-8588, Japan

${ }^{4}$ Laboratoire AIM, CEA/DSM-CNRS-Université Paris Diderot, IRFU/Service d'Astrophysique, CEA Saclay, F-91191 Gif-sur-Yvette, France

${ }^{5}$ Department of Physics and Astronomy, Rice University, 6100 Main Street, Houston, TX 77005, USA

${ }^{6}$ I. Physikalisches Institut, Universität zu Köln, Zülpicher Str. 77, D-50937 Köln, Germany

${ }^{7}$ California Institute of Technology, Cahill Center for Astronomy and Astrophysics 249-17, Pasadena, CA 91125, USA

${ }^{8}$ Haystack Observatory, Massachusetts Institute of Technology, 99 Millstone Road, Westford, MA 01886, USA

${ }_{9}$ Max-Planck-Institut für Radioastronomie, Auf dem Hügel 69, D-53121 Bonn, Germany

${ }_{11}^{10}$ Max-Planck-Institut für extraterrestrische Physik, Giessenbachstrasse 1, D-85748 Garching, Germany

${ }^{11}$ Department of Physics and Astronomy, Stony Brook University, Stony Brook, NY 11794-3800, USA

12 Department of Astrophysical and Planetary Sciences, University of Colorado, Boulder, CO, USA

${ }^{13}$ Sorbonne Université, Observatoire de Paris, Université PSL, CNRS, LERMA, F-75014, Paris, France

${ }_{14}$ California Institute of Technology, Cahill Center for Astronomy and Astrophysics 301-17, Pasadena, CA 91125, USA

${ }^{15}$ Institut de Ciències del Cosmos, Universitat de Barcelona, IEEC-UB, Martí i Franquès 1, E08028 Barcelona, Spain ${ }^{16}$ ICREA, Pg. Lluís Companys 23, E-08010 Barcelona, Spain

${ }^{17}$ Universität Heidelberg, Zentrum für Astronomie, Albert-Ueberle-Str. 2, D-69120 Heidelberg, Germany

${ }^{18}$ Universität Heidelberg, Interdisziplinäres Zentrum für Wissenschaftliches Rechnen, INF 205, D-69120 Heidelberg, Germany

${ }^{19}$ East Asian Observatory, 660 N. A'ohoku Place, Hilo, HI 96720, USA

${ }^{20}$ Harvard-Smithsonian Center for Astrophysics, 60 Garden Street, MS 42, Cambridge, MA 02138, USA

21 Jet Propulsion Laboratory, California Institute of Technology, 4800 Oak Grove Drive, Pasadena, CA 91109, USA

${ }^{22}$ Department of Earth, Space, and Environmental Sciences, College of the Canyons, Santa Clarita, CA 91355, USA ${ }^{23}$ Astronomy Department, University of Maryland, College Park, MD 20742, USA

${ }^{24}$ Department of Astronomy, The University of Tokyo, 7-3-1 Hongo Bunkyo, Tokyo 113-0033, Japan

25 National Radio Astronomy Observatory, 1003 Lopezville Road, Socorro, NM 87801, USA

${ }^{26}$ Institute for Theory and Computation, Harvard-Smithsonian Center for Astrophysics, 60 Garden St., Perkin Lab 240, Cambridge, MA 02138, USA

27 Jodrell Bank Centre for Astrophysics, School of Physics and Astronomy, University of Manchester, Oxford Road, Manchester M13 9PL, UK

28 Department of Physics, Niigata University, 8050 Ikarashi-2, Niigata 950-2181, Japan

29 Japan Women's University, 2-8-1 Mejirodai, Bunkyo-ku, Tokyo 112-8681, Japan

${ }^{30}$ College of Science, Ibaraki University, 2-1-1 Bunkyo, Mito, Ibaraki 310-8512, Japan

Received 2018 February 6; revised 2018 March 28; accepted 2018 March 29; published 2018 May 17

\begin{abstract}
We present the first results from a new, high-resolution ${ }^{12} \mathrm{CO}(1-0),{ }^{13} \mathrm{CO}(1-0)$, and $\mathrm{C}^{18} \mathrm{O}(1-0)$ molecular-line survey of the Orion A cloud, hereafter referred to as the CARMA-NRO Orion Survey. CARMA observations have been combined with single-dish data from the Nobeyama $45 \mathrm{~m}$ telescope to provide extended images at about $0.01 \mathrm{pc}$ resolution, with a dynamic range of approximately 1200 in spatial scale. Here we describe the practical details of the data combination in $u v$ space, including flux scale matching, the conversion of single-dish data to visibilities, and joint deconvolution of single-dish and interferometric data. A $\Delta$-variance analysis indicates that no artifacts are caused by combining data from the two instruments. Initial analysis of the data cubes, including moment maps, average spectra, channel maps, position-velocity diagrams, excitation temperature, column density, and line ratio maps, provides evidence of complex and interesting structures such as filaments, bipolar outflows, shells, bubbles, and photo-eroded pillars. The implications for star formation processes are profound, and follow-up scientific studies by the CARMA-NRO Orion team are now underway. We plan to make all the data products described here generally accessible; some are already available at https://dataverse.harvard.edu/dataverse/CARMA-NRO-Orion.
\end{abstract}

Key words: ISM: clouds - ISM: jets and outflows - ISM: kinematics and dynamics - ISM: structure - photondominated region (PDR) - stars: formation

Supporting material: animations, figure sets

\section{Introduction}

Star formation (SF) in the Milky Way takes place in giant molecular clouds (GMCs) that can contain $10^{5} M_{\odot}$ of gas. As described in a recent review by Heyer \& Dame (2015), a complete understanding of the properties of these clouds and their overall role in SF has been a major goal for over $50 \mathrm{yr}$. Nevertheless, many questions remain. A key issue is rooted in the high spatial dynamic range of SF processes, where the span of physical scales runs from $\sim 10$ pc for GMCs to $\sim$ au for protostars. This dramatic contrast, a factor of $\sim 10^{6}$, demands 
high dynamic range observations. By combining molecular-line interferometer and single-dish observations, it is now possible to map extended areas of molecular clouds and simultaneously probe small-scale SF scenarios within the larger-scale structures of the GMC gas. The resulting high dynamic range images can provide the comprehensive picture of SF in nearby GMCs that is critical to improving our understanding of the many processes involved in the formation of stars. Topics that can be more fully addressed include the roles of turbulence and magnetic fields in SF, the overall efficiency of the SF process, and the effects of feedback mechanisms.

For example, it is well known that GMCs are highly turbulent (e.g., Mac Low \& Klessen 2004; McKee \& Ostriker 2007; Padoan et al. 2014; Klessen \& Glover 2016). A variety of explanations for the observed filamentary structures ( $\mathrm{Li}$ et al. 2018) and velocity fields (Hennebelle \& Falgarone 2012), as well as signatures such as the mass surface density probability distribution function ( $\Sigma$-PDF; Federrath \& Banerjee 2015), have been put forward based on numerical simulations (also see Federrath et al. 2010). Large-scale mapping of the molecular gas enables studies of turbulence on all scales and provides statistically meaningful results for comparison with these simulations. Other signatures, such as the effect of magnetic fields on large-scale gas kinematics, may also be examined. Most importantly, the high dynamic range images provide much-needed information for GMCs with active SF across the whole mass spectrum.

Likewise, large-scale molecular gas maps encompassing a variety of cloud environments make it possible to study the formation and evolution of young stars in detail, from the first stages of dense core formation through the various protostellar phases. In fact, at any given time, only a few percent of the cloud mass is of sufficiently high density to form stars (Kennicutt \& Evans 2012). Most of the mass resides in low-density quiescent areas (see, e.g., Krumholz \& McKee 2005). Understanding how the cloud material transitions from a low- to a high-density state, and why such a small fraction of the mass is confined to dense cores, is critical to determining the physical processes that control the SF rate in molecular clouds. Numerical simulations suggest that turbulence, magnetic fields, and feedback effects can all cause inefficient SF (e.g., Nakamura \& Li 2007; Burkhart et al. 2009; Collins et al. 2012; Padoan et al. 2012, 2017; Krumholz et al. 2014; Federrath 2015; Li et al. 2017; Cunningham et al. 2018). Detailed large-scale images of various molecular tracers can complement these simulations. In particular, CO isotopologue maps can inform studies of feedback effects such as stellar winds (Arce et al. 2011; Li et al. 2015), outflows (Arce et al. 2010; Ginsburg et al. 2011; Narayanan et al. 2012), radiative heating (Goicoechea et al. 2015), and supernovae (Chen et al. 2017).

The Orion A cloud provides an ideal laboratory to map an extended molecular cloud in detail and address many of the scientific questions raised above. An active, star-forming region where both high- and low-mass young stellar objects are observed, the Orion A cloud encompasses a range of starforming environments (Bally 2008). It is a huge mass reservoir $\left(10^{5} M_{\odot}\right.$; Bally 2008) displaying a range of kinematic and dynamic characteristics and numerous features of feedback effects, including shocks, jets, outflows, bubbles, and photondominated regions (PDRs). Perhaps most importantly, at a distance of about $400 \mathrm{pc}$, it is the closest site of massive SF. As a result, high spatial dynamic range imaging of the molecular gas in Orion A will enable detailed comparison with a wide variety of numerical simulations covering physics and chemistry, low-mass SF to high-mass SF, and isolated and binary SF, as well as cluster formation.

Here we present the Combined Array for Research in Millimeter Astronomy (CARMA)-Nobeyama Radio Observatory (NRO) Orion Survey, our large-scale, high dynamic range imaging program for $\mathrm{CO}$ isotopologues in the Orion A cloud. To date, there have been a significant number of large-scale surveys of the Orion cloud at millimeter and far-infrared wavelengths. These, and their corresponding spatial resolutions, are listed in Table 1 for comparison purposes. Using CARMA, we have mapped a $2 \mathrm{deg}^{2}$ region of the cloud in ${ }^{12} \mathrm{CO}$ $J=1-0,{ }^{13} \mathrm{CO} J=1-0, \mathrm{C}^{18} \mathrm{O} J=1-0, \mathrm{CN} 1-0, J=3 / 2-1 / 2$, SO 2(3)-1(2), CS $J=2-1$, and $3 \mathrm{~mm}$ continuum emission. The results for the millimeter dust continuum and other molecular species will be presented in a future paper. The same region was mapped in ${ }^{12} \mathrm{CO} J=1-0,{ }^{13} \mathrm{CO} J=1-0$, and $\mathrm{C}^{18} \mathrm{O}$ $J=1-0$ using the NRO $45 \mathrm{~m}$ telescope (NRO45). Our broad goal was to combine the CARMA and NRO45 images and trace the gas kinematics on scales from $\sim 16 \mathrm{pc}$ down to about $0.01 \mathrm{pc}$, corresponding to $\sim 2000 \mathrm{au}$ at our adopted distance of $400 \mathrm{pc}^{31}$ The resulting images are the first molecular-line maps of a GMC at such a large spatial dynamic range $(\sim 1200)$ and with a resolution that is sufficient to study even protostellar envelopes in detail.

Bally et al. (1987) mapped the Orion A cloud in ${ }^{13} \mathrm{CO}$ $J=1-0$ with the Bell Lab $7 \mathrm{~m}$ telescope. Their map, at a resolution of $97^{\prime \prime}$, covers a larger region than our map, including the integral-shaped filament (ISF) and the entire L1641 cloud (i.e., from about decl. $=-4^{\circ}$ to $-9^{\circ}$ ). Ripple et al. (2013) mapped the Orion A cloud in ${ }^{12} \mathrm{CO} J=1-0$ and ${ }^{13} \mathrm{CO}$ $J=1-0$ with the Five College Radio Astronomy Observatory (FCRAO) $14 \mathrm{~m}$ telescope at a resolution of $46^{\prime \prime}$. These maps of Orion A have significantly lower resolution (by a factor of 5-10) compared to the maps presented here. Table 1 lists these and other selected large-scale maps and surveys of the Orion A cloud observed at different wavelengths.

\section{Observations}

\subsection{CARMA}

The CARMA 23-element millimeter wavelength array comprised six $10 \mathrm{~m}$ diameter antennas, nine $6 \mathrm{~m}$ antennas, and eight $3.5 \mathrm{~m}$ antennas. For this survey, observations were obtained with the 10 and $6 \mathrm{~m}$ antennas in the CARMA "D" and "E" configurations to provide $u v$ coverage encompassing 7-104 m (2.5-40 k $\lambda$, where $\lambda=2.6 \mathrm{~mm}$ at $115 \mathrm{GHz})$, corresponding to angular scales of $5^{\prime \prime}-70^{\prime \prime}$.

The region surveyed encompasses an area of approximately $2 \mathrm{deg}^{2}$ toward the Orion A cloud, bounded by the OMC-3 in the north and L1641-C in the south. This was divided into 181 subfields, each of $6^{\prime} \times 6^{\prime}$ size. Each subfield was covered in 126 pointings observed with a Nyquist sampling in a hexagonal grid, for a total of 22,806 pointings over $650 \mathrm{hr}$. For one pass through each subfield, the integration time was typically $15-20 \mathrm{~s}$ per pointing. For each array configuration, most subfields were observed three times at hour angles spaced by 2 hr to maximize the $u, v$ coverage. Hour-angle coverage in the two configurations was shifted by $1 \mathrm{hr}$, such that most subfields were observed six times at six different hour angles.

\footnotetext{
${ }^{31}$ Recent estimates of the distance to the Orion Nebula Cluster (ONC) lie between 414 pc (Menten et al. 2007) and 388 pc (Kounkel et al. 2017).
} 
Table 1

Selected Multiwavelength Surveys in the Orion A Cloud

\begin{tabular}{|c|c|c|c|}
\hline Telescope/Survey & Data Type & Resolution & Key Reference \\
\hline Bell Lab 7 m & ${ }^{13} \mathrm{CO}(1-0)$ & $97^{\prime \prime}$ & Bally et al. (1987) \\
\hline JCMT & $450 \mu \mathrm{m}, 850 \mu \mathrm{m}$ & $7 ! 5,14^{\prime \prime}$ & Johnstone \& Bally (1999) \\
\hline Harvard-CfA $1.2 \mathrm{~m}$ & ${ }^{12} \mathrm{CO}(1-0)$ & $8 ! 4$ & Wilson et al. (2005) \\
\hline $\mathrm{NRO} 45 \mathrm{~m}$ & $\mathrm{H}^{13} \mathrm{CO}^{+}(1-0)$ & $21^{\prime \prime}$ & Ikeda et al. (2007) \\
\hline ASTE, NRO $45 \mathrm{~m}$ & $1.1 \mathrm{~mm},{ }^{12} \mathrm{CO}(1-0)$ & $36^{\prime \prime}, 21^{\prime \prime}$ & Shimajiri et al. (2011) \\
\hline Spitzer/Spitzer Orion & MIR 3-24 $\mu \mathrm{m}$ & $2^{\prime \prime}-5^{\prime \prime}$ & Megeath et al. (2012) \\
\hline JCMT/GBS & ${ }^{13} \mathrm{CO}(3-2), \mathrm{C}^{18} \mathrm{O}(3-2)$ & $17^{\prime \prime}$ & Buckle et al. (2012) \\
\hline FCRAO $14 \mathrm{~m}$ & ${ }^{12} \mathrm{CO}(1-0),{ }^{13} \mathrm{CO}(1-0)$ & $46^{\prime \prime}$ & Ripple et al. (2013) \\
\hline Herschel/HOPS & FIR 70-160 $\mu \mathrm{m}$ & $5^{\prime \prime}-12^{\prime \prime}$ & Fischer et al. (2013) \\
\hline Herschel/HGBS & FIR 70-500 $\mu \mathrm{m}$ & $10^{\prime \prime}-37^{\prime \prime}$ & Roy et al. (2013) \\
\hline IRAM 30 m & ${ }^{12} \mathrm{CO}(2-1),{ }^{13} \mathrm{CO}(2-1)$ & $11^{\prime \prime}$ & Berné et al. (2014) \\
\hline Herschel-Planck dust & NIR, FIR, mm & $2^{\prime \prime}-5^{\prime}$ & Lombardi et al. (2014) \\
\hline JCMT/GBS & $450 \mu \mathrm{m}, 850 \mu \mathrm{m}$ & $8^{\prime \prime}, 13^{\prime \prime}$ & Salji et al. (2015) \\
\hline VISTA/VISION & NIR $0.85-2.4 \mu \mathrm{m}$ & $0 ! .8$ & Meingast et al. (2016) \\
\hline APO $2.5 \mathrm{~m} / \mathrm{IN}-\mathrm{SYNC}$ & NIR $1.5-1.6 \mu \mathrm{m}$ & $1 ! 6$ & Da Rio et al. (2016) \\
\hline GBT/GAS & $\mathrm{NH}_{3}(1,1)(2,2)(3,3)$ & $32^{\prime \prime}$ & Kirk et al. (2017) \\
\hline JCMT/BISTRO & $850 \mu \mathrm{m}$ polarization & $14^{\prime \prime}$ & Ward-Thompson et al. (2017) \\
\hline CARMA+NRO 45 m/CARMA-NRO Orion & ${ }^{12} \mathrm{CO}(1-0),{ }^{13} \mathrm{CO}(1-0), \mathrm{C}^{18} \mathrm{O}(1-0)$ & $8^{\prime \prime}$ & This paper \\
\hline
\end{tabular}

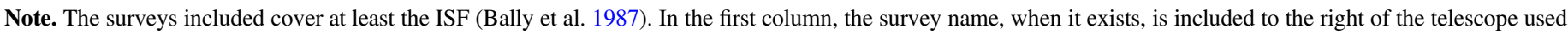
for the survey.

Observations were carried out between 2013 and 2015 as a CARMA Key Project. The receiver was tuned to a local oscillator (LO) frequency of $109.41 \mathrm{GHz}$, and the correlator was configured to cover the ${ }^{12} \mathrm{CO} J=1-0,{ }^{13} \mathrm{CO} J=1-0, \mathrm{C}^{18} \mathrm{O} J=1-0, \mathrm{CN}$ $1-0, J=3 / 2-1 / 2$, and SO 2(3)-1(2) lines in the upper sideband (USB) and CS $J=2-1$ in the lower sideband (LSB). The correlator windows for ${ }^{12} \mathrm{CO} J=1-0$ and $\mathrm{CN}$ used a bandwidth of $31 \mathrm{MHz}\left(81 \mathrm{~km} \mathrm{~s}^{-1}\right)$ with a spectral resolution of $98 \mathrm{kHz}$ $\left(\sim 0.25 \mathrm{~km} \mathrm{~s}^{-1}\right)$. The correlator windows for the remaining spectral lines had bandwidths of $8 \mathrm{MHz}\left(\sim 21 \mathrm{~km} \mathrm{~s}^{-1}\right)$ and spectral resolutions of $24 \mathrm{kHz}\left(\sim 0.067 \mathrm{~km} \mathrm{~s}^{-1}\right)$. The correlator setup also included two $500 \mathrm{MHz}$ bandwidth windows centered at 110.8 and $111.3 \mathrm{GHz}$ in USB, with the image sideband centered at 103.0 and $102.5 \mathrm{GHz}$, for a total of $2 \mathrm{GHz}$ continuum bandwidth. In this paper, only the ${ }^{12} \mathrm{CO} J=1-0,{ }^{13} \mathrm{CO} J=1-0$, and $\mathrm{C}^{18} \mathrm{O} J=1-0$ CARMA data are presented.

Calibration of the bandpass for each spectral window relied primarily on observations of 3C84, but the quasars 0423-013 and $0532+075$ were also used. Uranus was the primary flux calibrator. When it was not available, Mars or observations of 3C84 calibrated from prior observations were used. Gain calibration relied on observations of $0532+075$ every $\sim 20$ minutes. The MIRIAD (Sault et al. 1995) task mossdi was used to create maps from the CARMA observations (with a Briggs weighting of 0.5 ) with an angular resolution of $\sim 7^{\prime \prime}$ and sensitivity of $0.62 \mathrm{Jy} \mathrm{beam}^{-1}$ in a $0.25 \mathrm{~km} \mathrm{~s}^{-1}$ channel for ${ }^{12} \mathrm{CO}$ and 0.50 Jy beam ${ }^{-1}$ in a $0.10 \mathrm{~km} \mathrm{~s}^{-1}$ channel for ${ }^{13} \mathrm{CO}$ and $\mathrm{C}^{18} \mathrm{O}$.

\section{2. $\mathrm{NRO45}$}

NRO45 mapping observations of the Orion A cloud in the ${ }^{12} \mathrm{CO}(1-0),{ }^{13} \mathrm{CO}(1-0)$, and $\mathrm{C}^{18} \mathrm{O}(1-0)$ lines were made using two receivers: (1) BEARS, a 25-beam receiver, and (2) FOREST, a newer, four-beam dual polarization, sidebandseparating SIS receiver (Minamidani et al. 2016). The telescope beam size is $\sim 15^{\prime \prime}$ at $115 \mathrm{GHz}$, and the main-beam efficiency $\eta_{\mathrm{MB}}$ is $\sim 27 \%-43 \%$.
BEARS observations of the Orion A cloud in ${ }^{12} \mathrm{CO}(1-0)$ were acquired between 2007 December and 2009 January. The isotopologues ${ }^{13} \mathrm{CO}(1-0)$ and $\mathrm{C}^{18} \mathrm{O}(1-0)$ were observed between 2013 May and 2014 January. Some of these data have already been published (Shimajiri et al. 2011, 2014, 2015a; Nakamura et al. 2012). A combination of 25 sets of 1024-channel autocorrelators (one for each receiver) with $32 \mathrm{MHz}$ bandwidth provided a frequency resolution of $31.25 \mathrm{kHz}$ (Sorai et al. 2000), corresponding to $\sim 0.1 \mathrm{~km} \mathrm{~s}^{-1}$ velocity resolution at $115 \mathrm{GHz}$. Standard on-the-fly (OTF) mapping techniques with off position (R.A.J2000, decl.J2000 $)=\left(5^{\mathrm{h}} 29^{\mathrm{m}} 00^{\mathrm{s}} \cdot 0,-5^{\circ} 25^{\prime} 30\right.$ !" 0$)$ were used to produce images of the extended cloud. To minimize any adverse effects due to the process, separate OTF maps were obtained by scanning in the R.A. and decl. directions. Pointing accuracy was better than $3^{\prime \prime}$ throughout the observations.

NRO45 mapping observations of the Orion A cloud using the FOREST receiver were carried out between 2014 December and 2017 February. Until 2016 December, the ${ }^{12} \mathrm{CO}(1-0),{ }^{13} \mathrm{CO}(1-0)$, and $\mathrm{C}^{18} \mathrm{O}(1-0)$ molecular lines were observed simultaneously. Thereafter, due to a change in the receiver setting, only ${ }^{13} \mathrm{CO}(1-0)$ and $\mathrm{C}^{18} \mathrm{O}(1-0)$ were included.

The SAM45 spectrometer provided a bandwidth of $63 \mathrm{MHz}$ and frequency resolution of $15.26 \mathrm{kHz}$, corresponding to a velocity resolution of $\sim 0.04 \mathrm{~km} \mathrm{~s}^{-1}$. The line intensity scale was derived from direct comparison with the ${ }^{12} \mathrm{CO}(1-0)$ and ${ }^{13} \mathrm{CO}(1-0) T_{\mathrm{mb}}$ maps obtained by the BEARS receiver.

During the FOREST observations, we mapped a small area of the OMC-2/FIR 4 region (the strongest dust continuum emission in the OMC-2/3 region; Shimajiri et al. 2008, 2015a, $2015 \mathrm{~b})$ two or three times per day. We scaled the FOREST $\left(T_{\mathrm{a}}^{*}\right)$ to the BEARS $\left(T_{\mathrm{mb}}\right)$ by comparing the FOREST intensity with the BEARS intensity in the OMC-2/FIR 4 region. As for the BEARS observations, the FOREST observations were obtained using the OTF mapping technique (with the same reference position), and the pointing accuracy was better than $3^{\prime \prime}$.

To produce the final NRO45 map, we combined the BEARS and FOREST observations. As a convolution 
function, we adopted a spheroidal function with a spatial grid size of $7 !$ " 5 , resulting in the final effective angular resolution of $\sim 22^{\prime \prime}$.

As a check on the adopted intensity scale for the NRO45 data, comparisons were made with published maps of the Orion A cloud from the FCRAO $14 \mathrm{~m}$ telescope (Ripple et al. 2013) and the Bell Lab $7 \mathrm{~m}$ telescope (Bally et al. 1987). We also compared the ${ }^{13} \mathrm{CO}(1-0) \mathrm{NRO} 45$ data with recent observations conducted with the IRAM 30 m telescope (projects 030-15 and 127-15, Á. Sanchez-Monge 2018, private communication). To ensure a meaningful comparison, the NRO45 image cube was convolved with a Gaussian kernel using the MIRIAD convol command so that there was a good match between the spatial and velocity resolutions of the other data sets. We find that the NRO45 intensity scale is about $15 \%-25 \%$ higher than those in the FCRAO and Bell Lab maps and within $15 \%$ with respect to the IRAM $30 \mathrm{~m}$ data. However, this level of discrepancy is not unusual for single-dish observations due to calibration uncertainties.

\section{Combining NRO45 and CARMA Data}

Here we provide the practical details of how we combined the single-dish NRO45 and CARMA interferometer observations in the $u v$ plane, following the procedure outlined in Koda et al. (2011). The data included in the combination range from zerospacing fluxes to measurements on the maximum baselines provided by CARMA. This is especially important for regions with such high spatial dynamic range as the Orion A cloud.

\subsection{Relative Flux Scale}

The first step in combining the NRO45 and CARMA data is to establish the relative flux scale factor, $f \equiv F_{\mathrm{CARMA}} / F_{\mathrm{NRO} 45}$, between the interferometer and single-dish maps. The NRO45 telescope is treated as an interferometer with baselines ranging from 0 to $45 \mathrm{~m}$, corresponding to $u v$ distances of $0-17 \mathrm{k} \lambda$. For reference, the complete CARMA data set encompasses baselines from 3 to $40 \mathrm{k} \lambda$. The factor $f$ is determined by selecting visibilities in the $u v$ space where the two data sets overlap and comparing the respective fluxes. In cases where there are deviations from the canonical value of $f=1$, the interferometer flux is adopted because it is typically based on more frequent and more reliable calibration observations (Koda et al. 2011; Plunkett et al. 2013).

In practical terms, we are modeling the NRO45 image so that it appears to have been observed by a subset of the CARMA baselines, i.e., the range of baselines included in both maps. This requires some editing of each data set so that only regions with significant emission in both single-dish and interferometer images are included. Since the NRO45 sensitivity on baselines longer than $6 \mathrm{k} \lambda$ is relatively low (see Figure 5 in Koda et al. 2011), the CARMA data set was limited to observations from the $6 \mathrm{~m}$ antenna pairs with baselines between 3 and $6 \mathrm{k} \lambda$. The velocity channel comparisons were similarly constrained. CARMA dirty images of these "subregions" were produced by Fourier transforming the resulting visibilities using MIRIAD.

For the NRO45 data set, the intensity scale was converted to Jy beam $^{-1}$ using Equation (1) (where $\Omega_{\mathrm{mb}}=1.133^{*} \mathrm{FWHM}^{2}$ with FWHM in radians):

$$
S_{\nu}=T_{\mathrm{mb}} \frac{2 k \Omega_{\mathrm{mb}}}{\lambda^{2}}
$$

The resulting image was then regridded to match the CARMA map in terms of pixel size and channel width, as well as the total number of pixels and channels. It was also deconvolved using the original beam $\left(\sim 22^{\prime \prime}\right)$. This produced a fair representation of the NRO45 sky brightness distribution to be modeled as if it had been observed with the same $u v$ sampling as the modified CARMA map. To achieve this, the edited NRO45 image was broken down (demosaicked) to correspond to the different pointings that produced the CARMA mosaic map of the same area using the MIRIAD task demos.

As part of this "demosaicking" process, the response of a primary beam was included at each of the pointings to compensate for the fact that the MIRIAD task invert that is used later to produce a dirty image from the visibility data automatically corrects for the primary beam response (see Koda et al. 2011 for details). Each pointing from the demosaicking procedure for NRO45 was modeled based on the visibility distribution of the modified CARMA map described above (using the MIRIAD task uvmodel). The visibilities generated for each pointing were then merged, and the combined set was Fourier transformed to produce a dirty image of the modified NRO45 data set (using invert). As a result, each dirty image is constructed from an identical set of baselines selected from the CARMA visibility data between 3 and $6 \mathrm{k} \lambda$ and has the same dirty beam size.

Ratios of intensities were determined for each pixel in the modified CARMA and NRO45 dirty maps with a signal-tonoise ratio greater than 20 . Plots of the distribution of the ratio for different regions and channels show a consistent Gaussianlike distribution with an average peak value of about 1.6-1.8 for ${ }^{12} \mathrm{CO}$ and $1.5-1.6$ for ${ }^{13} \mathrm{CO}$ and $\mathrm{C}^{18} \mathrm{O}$. We note that the $\mathrm{C}^{18} \mathrm{O}(1-0)$ ratio was derived from integrated intensity maps since the emission is relatively weak, especially when the baseline range is constrained to $3-6 \mathrm{k} \lambda$. Adopted flux scale factors are 1.6 for ${ }^{12} \mathrm{CO}(1-0)$ and 1.5 for ${ }^{13} \mathrm{CO}(1-0)$ and $\mathrm{C}^{18} \mathrm{O}(1-0)$. These values are unexpected in that they are much higher than the canonical value of $f=1$. Consequently, we have carefully reexamined the flux calibration results and all assumptions and procedures used in deriving this factor.

A comparison of our CARMA intensities with other millimeter-wave interferometer measurements can help assure the accuracy of our data calibration. For example, we considered the ${ }^{12} \mathrm{CO}(1-0)$ and ${ }^{13} \mathrm{CO}(1-0)$ CARMA data obtained by Tobin et al. (2016) of a region in Orion in the southern part of our map. Both data sets were edited to ensure as much similarity as possible between the baselines involved, and bright emission structures were compared. We found the measured intensities to be consistent within the noise. A second comparison used unpublished ${ }^{12} \mathrm{CO}(1-0)$ observations from the ALMA $7 \mathrm{~m}$ dishes, the Atacama Compact Array (ACA; ALMA project 2016.1.01123.S, A. Hacar 2018, private communication). We modeled the CARMA measurements to appear as if they were observed with the baseline configuration of the ACA. Spectra and intensities in different regions of the resulting CARMA cube are also consistent with the ACA data. Finally, we used the Common Astronomy Software Applications (CASA) data reduction package (McMullin et al. 2007) to simulate ACA observations of the NRO45 image and derived a similarly high flux scale factor between NRO45 and the ALMA data. A similar value of $f$ also resulted when conducting similar tests in MIRIAD with the ACA data. 
Tests of the various procedures involved in our derivation of $f$ were also carried out. In particular, we repeated the procedures described using multiple regions and channels and constraining baselines to different $u v$ ranges (e.g., 3-4, 4-5, $5-6$, and $3-5 \mathrm{k} \lambda$ ). We also introduced different combinations of the 6 and $10 \mathrm{~m}$ dishes (i.e., different CARMA antenna pairs). In all cases, similar values of the scale factor were obtained. To ensure that the flux factor derivation is self-consistent, we also compared the CARMA image with "itself." Specifically, we smoothed the CARMA cleaned image to match the NRO45 resolution and then used the same script to compare the smoothed CARMA image with the original image. The resulting flux "ratio" was 1 , as expected. We were able to reproduce the original NRO45 data to within $3 \%$ by imaging the modeled NRO45 visibilities, confirming the validity of the deconvolution procedures. Using the immerge command in MIRIAD, we again determined the flux scale factor and found values of $\sim 1.6$ for ${ }^{12} \mathrm{CO}$ in various subregions.

\subsection{Combining the Matched Data Sets}

With the NRO45 and CARMA fluxes matched and the NRO45 observations treated as short spacing measurements in the larger CARMA $u v$ plane, combining the two data sets is already more straightforward. The CARMA data set requires no further overall modification, and a CARMA-only dirty map can be immediately constructed. For this preliminary map, we used the complete set of visibilities from the region mapped by CARMA but restricted to the velocity range $0-16.7 \mathrm{~km} \mathrm{~s}^{-1}$. Velocity channel widths are $0.25 \mathrm{~km} \mathrm{~s}^{-1}$ for ${ }^{12} \mathrm{CO}(1-0)$ and $0.22 \mathrm{~km} \mathrm{~s}^{-1}$ for both ${ }^{13} \mathrm{CO}(1-0)$ and $\mathrm{C}^{18} \mathrm{O}(1-0)$. For each pointing in the mosaics, we adopted a cell size of $2^{\prime \prime}$ and an image size of 95 cells, more than twice the size of the synthesized beam of the CARMA $6 \mathrm{~m}$ telescopes. These choices were largely dictated by our imaging requirements, i.e., pixel size $\sim 1 / 5-1 / 3 \times$ synthesized beam and image size $>2 \times$ field of view. Smaller cell sizes or larger image sizes require considerably more computing resources in terms of memory and time. The resulting CARMA-only dirty map was produced with the MIRIAD invert task, adopting a robust number of 0.5 and options = mosaic, double.

By contrast, a few more steps are necessary to prepare the modified and flux-corrected NRO45 visibilities for combination because we will work on the entire NRO45 image cube in order to fill the $u v$ "hole" in the CARMA visibilities. First, the NRO45 cube was converted from main-beam temperature $T_{\mathrm{mb}}$ to flux density $S_{\nu}$ using Equation (1). Then, we multiplied the new cube by the flux scale factor (see Section 3.1). The maths task was used for the two steps. Next, we regridded the NRO45 image cube to match the pixel and channel numbers and sizes of the CARMA-only preliminary dirty image and deconvolved it with the NRO45 beam $\left(\sim 22^{\prime \prime}\right)$ using the MIRIAD command convol with options=divide. The demosaicking procedure described in Section 3.1 was then applied. In this way, we obtain visibilities at each pointing of the CARMA mosaic as if the observations had been made with a set of randomly generated baselines that follow a Gaussian distribution and mimic the response of the NRO45 telescope.

The distribution of baselines was generated using hkuvrandom, a modified version of the standard task uvrandom developed by J. Koda in MIRIAD 4.3.8. In effect, a Gaussian distribution of $u v$ points (baselines) with a standard deviation sdev is generated. This Gaussian is the Fourier transform of the NRO45 primary beam with a standard deviation of $3.5 \mathrm{k} \lambda$. Due to our slightly larger effective primary beam, $22^{\prime \prime}$, this is slightly smaller than the value $(3.9 \mathrm{k} \lambda)$ used in Koda et al. (2011).

The total number of points generated by hkuvrandom is given by the parameter $N_{\text {total }}$ (npoint in hkuvrandom). It depends largely on the value assumed for the NRO45 visibility integration ( $t_{\mathrm{vis}}$, inttime in hkuvrandom) and the requirement that the sensitivity per pixel in $u v$ space for NRO45 visibilities is similar to that for CARMA in their $u v$ overlap range, 3-6 k $\lambda$ (Koda et al. 2011). Following Koda et al. (2011), we adopt $t_{\mathrm{vis}}=0.01 \mathrm{~s}$. This is sufficiently low to ensure that $N_{\text {total }}$ is large enough to fill the $u v$ space. We adopted $N_{\text {total }} \sim 3000$. Due to the low sensitivity of NRO45 observations on baselines greater than $6 \mathrm{k} \lambda$, we flagged such generated baselines before we applied the uvmodel MIRIAD command to the demosaicked images and used the remaining generated baselines to produce a visibility file for each mosaic pointing. The file header for each file was modified to include the NRO45 system temperature, the weighting parameter jyperk (see Koda et al. 2011, Equations (14)-(15)), and our arbitrary $2^{\prime}$ Gaussian primary beam (chosen to be larger than the CARMA primary beams). Finally, we merged all of the modeled NRO45 visibility files together to combine with the CARMA visibilities.

Imaging of the combined data was carried out with the Yale high-performance computing (HPC) clusters, Grace and Farnam. ${ }^{32}$ We produced a dirty cube of the joint CARMA and NRO45 observations by Fourier transforming the combined visibilities using the task invert. This requires roughly $40 \mathrm{~GB}$ of memory. As before, we adopted a $2^{\prime \prime}$ pixel size, approximately $1 / 5-1 / 3$ of the final beam size, and an image size at each mosaic pointing of $\sim 95$ pixels. The invert process for the ${ }^{12} \mathrm{CO}$ cube $(\sim 2500 \times 4300$ pixels, 90 channels $)$ took roughly 1 day and $2.5 \mathrm{GHz}$ CPU. Each channel was cleaned separately using the mossdi2 task in MIRIAD. For this procedure, each channel took up $1 \mathrm{CPU}$ and roughly $30 \mathrm{~GB}$ of memory (less memory is needed for channels with lower emission). All channels can be cleaned simultaneously using multiple CPUs. The cleaning process, with the stopping threshold for clean set to 3 times the rms noise level, took at most 1 day for each channel. The beam size and position angle, channel width, and rms noise per channel of the resulting maps are given in Table 2 .

Figure 1 shows the entire mosaic footprint for the ${ }^{12} \mathrm{CO}$ combined image overlaid on an RGB false-color image from Spitzer (R. Gutermuth 2018, private communication). The footprints of the ${ }^{13} \mathrm{CO}$ and $\mathrm{C}^{18} \mathrm{O}$ combined images are progressively smaller (due to limited coverage in NRO45 observations). Most importantly, all the maps span slightly more than $2^{\circ}$, or about $14 \mathrm{pc}$, in decl. and encompass a variety of well-known star-forming regions in the Orion cloud.

Figure 2, a comparison of the ${ }^{12} \mathrm{CO}$ peak intensity maps of a subregion in the central Orion box (see Figure 1) based on NRO45, CARMA, and the combined NRO45+CARMA data sets, demonstrates the effectiveness of combining single-dish and interferometer observations. The $19^{\prime} \times 18^{\prime} .7(2.21 \times$ $2.17 \mathrm{pc})$ maps are centered on the dense part of the Orion Nebula Cluster (ONC; also known as the Huygens Region) and the Orion KL regions at R.A. $05^{\mathrm{h}} 35^{\mathrm{m}} 26^{\mathrm{s}} .6$, decl. $-05^{\circ} 20^{\prime} 44^{\prime \prime} \cdot 9$.

\footnotetext{
32 https://research.computing.yale.edu/services/high-performancecomputing
} 
Table 2

Final Sensitivity

\begin{tabular}{lrccc}
\hline \hline Transition & Beam & $\begin{array}{c}\mathrm{PA} \\
(\mathrm{deg})\end{array}$ & $\begin{array}{c}\Delta_{V} \\
\left(\mathrm{~km} \mathrm{~s}^{-1}\right)\end{array}$ & $\begin{array}{c}\sigma_{K} \\
(\mathrm{~K})\end{array}$ \\
\hline${ }^{12} \mathrm{CO}(1-0)$ & $10^{\prime \prime} \times 8^{\prime \prime}$ & -13 & 0.25 & 0.86 \\
${ }^{13} \mathrm{CO}(1-0)$ & $8^{\prime \prime} \times 6^{\prime \prime}$ & 10 & 0.22 & 0.64 \\
$\mathrm{C}^{18} \mathrm{O}(1-0)$ & $10^{\prime \prime} \times 8^{\prime \prime}$ & -0.4 & 0.22 & 0.47
\end{tabular}

Note. The rms per channel shown in the last column is the median value across the relevant cube.

There is a dramatic improvement in the image quality after the CARMA and NRO45 data are combined, with considerably more detailed structure visible over the extended subregion. It is worth noting that, over this subregion, CARMA recovers only about $20 \%$ of the NRO45 detected flux.

\subsection{Using $\Delta$-variance Analysis to Verify Data}

A $\Delta$-variance analysis (Stutzki et al. 1998; Ossenkopf et al. 2008) can also provide confidence in the quality of the final emission-line images. Such analysis readily reveals peculiarities and artifacts in the spatial distributions and, at the same time, can characterize the global scaling behavior and prominent spatial scales in the maps. In principle, the $\Delta$-variance spectrum measures the relative amount of structure on a given scale $s$ by convolving the map being considered with a radially symmetric top-down wavelet of that scale and computing the variance of the convolved map as a function of $s$. Each data point can be weighted by a significance value before selecting the statistics to be examined, e.g., variable noise in the data. Stutzki et al. (1998) showed that for rectangular maps with equal weights of all points, the $\Delta$-variance spectrum is equivalent to the power spectrum; the slope, $d$, of the $\Delta$-variance spectrum corresponds to a power spectral slope $\beta=d-2$.

A $\Delta$-variance analysis can also be applied to maps with irregular boundaries and data with variable uncertainties (Ossenkopf et al. 2008) to obtain statistics on the size dependence of all structures in the map. Here we applied the analysis to the integrated intensity maps for each spectral line and to maps of the intensity in the central velocity channel at $v=8.8 \mathrm{~km} \mathrm{~s}^{-1}$. For our statistical analysis, we weighted each data point by the inverse rms noise in the 16 channels at the velocity boundaries of the cubes.

Figure 3 shows the $\Delta$-variance spectra for the integrated intensity maps. Figure 4 shows the corresponding spectra for the channel maps. Both demonstrate that combining NRO45 and CARMA data introduced no obvious artifacts in the ${ }^{12} \mathrm{CO}$ and ${ }^{13} \mathrm{CO}$ maps. Both cubes show a perfect power law at scales around $22^{\prime \prime}$ the effective NRO45 beam size. For $\mathrm{C}^{18} \mathrm{O}$, the agreement was not as good. In addition, the $\Delta$-variance spectrum displays a peak at about $25^{\prime \prime}$, and an inspection of the $\mathrm{C}^{18} \mathrm{O}$ map indicates the presence of granular structure on that scale. Both results are likely attributable to overall lower signalto-noise in the $\mathrm{C}^{18} \mathrm{O}$ maps, greater noise in the NRO45 maps than in the CARMA maps, and accompanying issues with the cleaning process.

At lag scales below $0.13\left(8^{\prime \prime}\right)$, the $\Delta$-variance spectra of all maps are dominated by the natural limitations of the CARMA observations. Since the major axis of the synthesized CARMA beam is about $8^{\prime \prime}$, structures with smaller sizes will be blurred, reducing their contribution to the $\Delta$-variance spectrum. In contrast, $\Delta$-variance spectra tend to increase at the smallest lags, since the small-scale spectrum is dominated by observational noise that introduces excess power. This effect is particularly relevant to the central velocity channel maps, where the relative noise is higher than in the integrated intensity maps. The impact of the finite telescope beam can be seen when the spectra for the full combined data set are compared with those obtained from the NRO45 maps at the $22^{\prime \prime}$ resolution. Structural variations on scales up to about three beam widths are suppressed in the absence of the CARMA observations, as expected from computations by Bensch et al. (2001). At larger scales, the $\Delta$-variance spectra show no resolution dependence. Likewise, smoothing the full data set to the resolution of the $45 \mathrm{~m}$ telescope data reproduced the spectra obtained from the single-dish maps.

\section{Results}

\subsection{Gas Distribution}

Figures 5-7 show the integrated intensity maps ${ }^{33}$ for ${ }^{12} \mathrm{CO}(1-0),{ }^{13} \mathrm{CO}(1-0)$, and $\mathrm{C}^{18} \mathrm{O}(1-0)$, respectively, over the velocity range $2.5-15 \mathrm{~km} \mathrm{~s}^{-1}$. As already noted, the maps span about $2^{\circ}$ in decl. and encompass a range of star-forming regions. Each extends from OMC-3 and OMC-2, at decl. $\sim-5^{\circ}$, to the V380 Ori Group (NGC 1999) and the L1641-C region at nearly $\sim-7^{\circ}$. Several well-known regions are labeled. The relatively noisy patches that are noticeable around R.A. $05^{\mathrm{h}} 33^{\mathrm{m}} 25^{\mathrm{s}} .0$, particularly in the ${ }^{12} \mathrm{CO}(1-0)$ map, are due to limited NRO45 observing time and the resulting lower sensitivity. Other widespread, complex structures can also be seen. Some of these have been studied extensively, but many have barely been mentioned in the literature. Since we expect that numerous detailed studies and analyses will follow our dissemination of these data, we draw attention to only a few highlights of the new maps here.

The ONC is probably the most-studied region in the Orion A cloud (e.g., Figure 1 of Meingast et al. 2016). Not surprisingly, it appears as one of the most dramatic regions in all three of our molecular-line maps. In particular, the area enclosing the ONC is clearly seen as a cavity in the gas distribution, presumably due to clearing of molecular gas in the $\mathrm{H}$ II region that is powered by high-mass stars in the associated cluster (O'Dell et al. 2017). The cavity itself is bounded by the bright edges that are expected when ionization/dissociation fronts interact with the surrounding molecular cloud. The brightest edge, to the southeast, is commonly referred to as the Orion Bar (see, e.g., Pellegrini et al. 2009; Goicoechea et al. 2017). Another cavity in the molecular gas distribution is visible north of the ONC. This is produced by the H II region M43 (Thum et al. 1978; Shimajiri et al. 2011, 2013).

Further north, our maps show the molecular gas associated with the OMC-2 and OMC-3 star-forming regions. These host a wealth of phenomena associated with early SF and include protostars and outflows in a range of evolutionary states (Shimajiri et al. 2008, 2009; Megeath et al. 2012; LópezSepulcre et al. 2013; Stutz et al. 2013). Immediately to their west, a region of significantly lower intensity is particularly noticeable in the ${ }^{13} \mathrm{CO}$ map (Figure 6). This is bounded on its far side by a prominent arc of emission that continues

\footnotetext{
33 These and most other maps in this paper are available online at https:// dataverse.harvard.edu/dataverse/CARMA-NRO-Orion.
} 


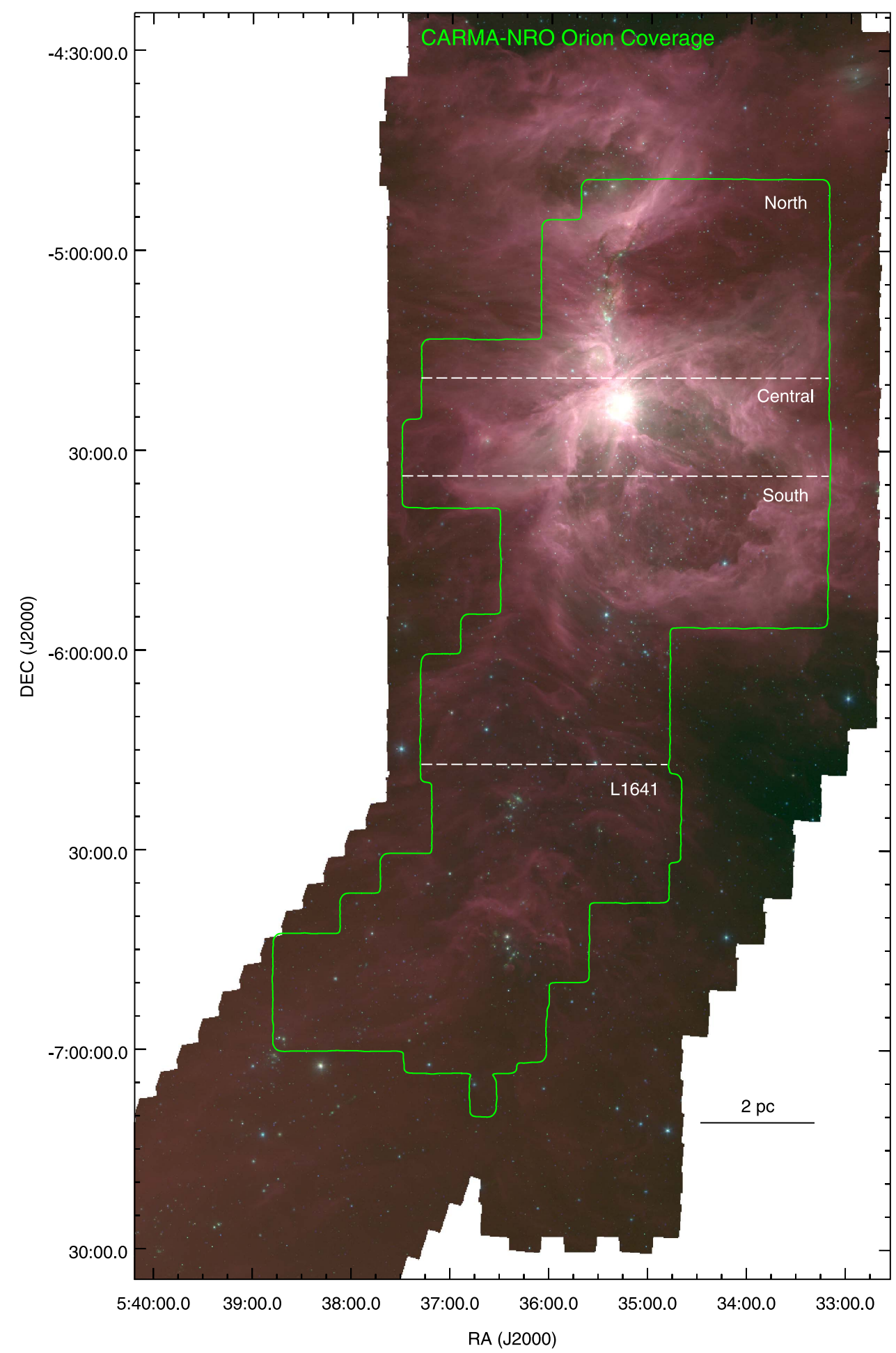

Figure 1. Footprint of the ${ }^{12} \mathrm{CO}$ CARMA-NRO Orion map (green solid lines) overlaid on a Spitzer/IRAC false-color RGB image based on $8 \mu \mathrm{m}$ (red), $4.5 \mu \mathrm{m}$ (green), and $3.4 \mu \mathrm{m}$ (blue) observations. White dashed lines designate four regions of interest (Feddersen et al. 2018) that will be used in Section 4.2 .

southward to OMC-4, suggesting the presence of an extended cavity-like structure. A similarly elongated, lower-intensity region is seen to the southeast of the ONC and Orion Bar. This depression is bounded on the east by structures that have the appearance of irradiated and elongated globules or pillars with bright edges. They can be discerned more clearly in the inset in Figure 5 and appear to be pointing toward the ionizing stars of the ONC, consistent with photo-erosion by the UV radiation emanating from the massive stars in the region.
One of the most striking structures in the Orion A cloud is the so-called ISF, first seen in lower-resolution maps (Bally et al. 1987). In Figure 5, this structure is also detected and stretches about $1^{\circ}$ from north to south (decl. $-5^{\circ}$ to $\sim-6^{\circ}$ ). As we discuss below, considerable filamentary structure on a variety of scales is especially clear in our ${ }^{13} \mathrm{CO}$ maps covering different velocity ranges and will provide a wealth of information for comparison with numerical simulations of molecular clouds (e.g., S. Suri et al. 2018, in preparation). 

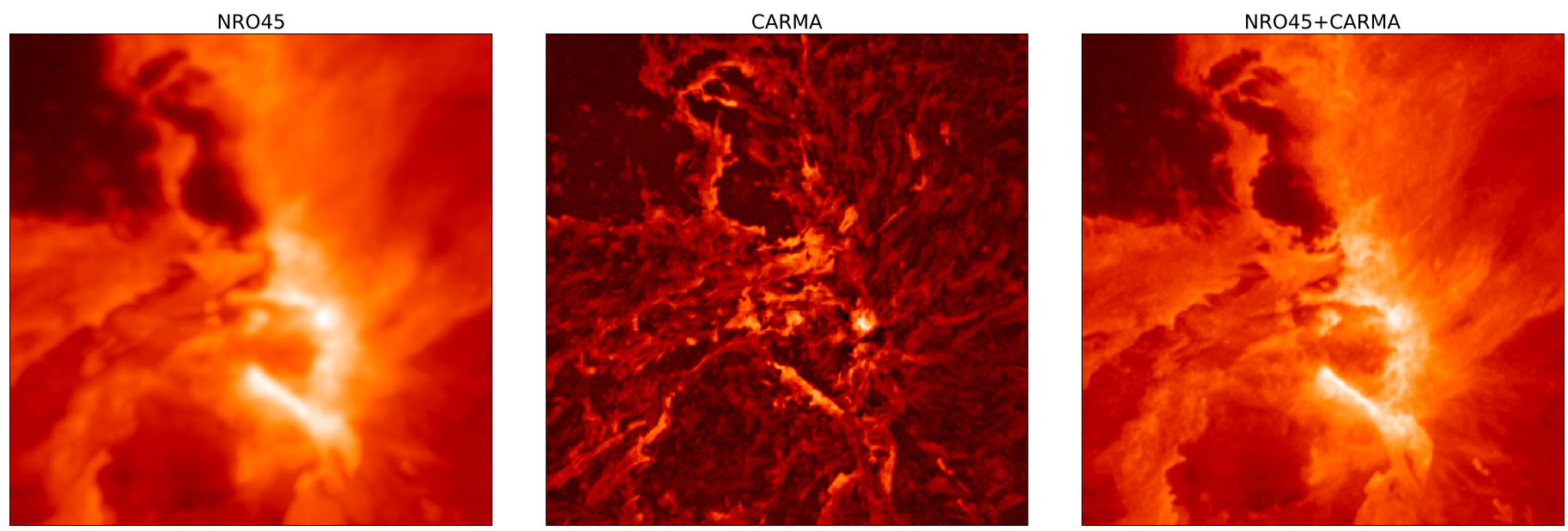

Figure 2. Sample comparison of the NRO45, CARMA, and combined maps. (Left) ${ }^{12} \mathrm{CO}$ peak intensity maps from NRO45, (middle) CARMA, and (right) the combined CARMA+NRO45 data sets. All panels show the same $19^{\prime} .0 \times 18^{\prime} .7(2.21 \mathrm{pc} \times 2.17 \mathrm{pc})$ area centered around the ONC and Orion KL region and includes the Orion Bar. The increased sharpness of the extended images when the CARMA observations are combined with those from NRO45 is immediately clear.

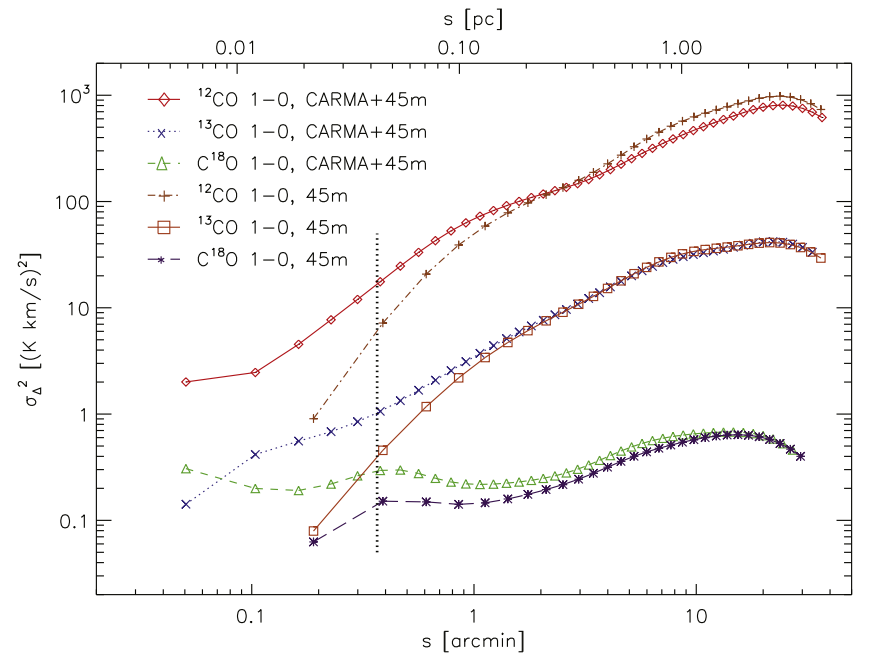

Figure 3. The $\Delta$-variance spectra for the ${ }^{12} \mathrm{CO}(1-0),{ }^{13} \mathrm{CO}(1-0)$, and $\mathrm{C}^{18} \mathrm{O}(1-0)$ integrated intensity maps from $\mathrm{NRO} 45$ and the combined (CARMA+NRO45) data set. On the $x$-axis, the lag indicates the scale measured by the wavelet size. The $y$-axis displays the variance of the structural fluctuations at each value of $x$ in units of the square of the measured map. The vertical dotted line indicates the NRO45 beam size.

The L1641 cloud lies (see Allen \& Davis 2008) at the south end of our maps. Our maps encompass the gas associated with the L1641-N and NGC 1999 clusters, while their southern borders coincide with the northern outskirts of the L1641-C cluster. This region of the Orion A cloud harbors no high-mass stars, but a few intermediate-mass objects are present, as are several groups of young low-mass stars. Again, there is considerable potential for a wide range of SF studies (e.g., H. Arce et al. 2018, in preparation).

\subsubsection{Cloud Structure from $\Delta$-variance Analysis}

The $\Delta$-variance spectrum of the integrated intensity ${ }^{13} \mathrm{CO}$ map (Figure 3) is an almost perfect power-law spectrum over all scales from $20^{\prime \prime}$ to $7^{\prime}$. The slope is 1.1 , corresponding to a power spectral index $\beta=3.1$. At larger scales, the spectrum becomes shallower, indicating a relative lack of structure. For the corresponding channel map (Figure 4), the inertial range starts only at $25^{\prime \prime}$ due to effects of higher noise, while the powerlaw slope is somewhat shallower, $\beta=3.0$ (see discussions

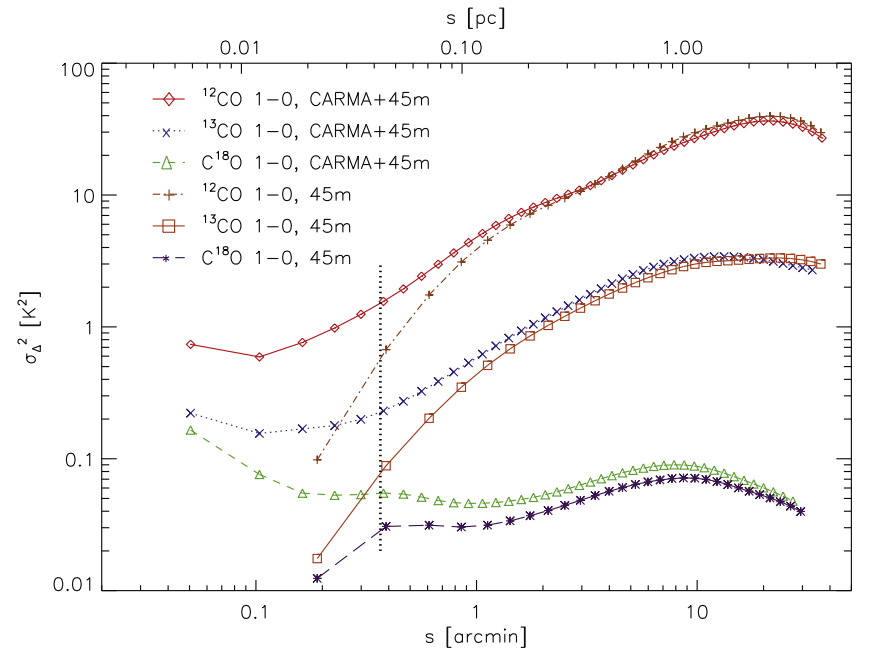

Figure 4. The $\Delta$-variance spectra for maps of the central velocity channel intensity $\left(v=8.8 \mathrm{~km} \mathrm{~s}^{-1}\right.$ with channel width $\Delta v=0.25$ for ${ }^{12} \mathrm{CO}$ and $0.22 \mathrm{~km} \mathrm{~s}^{-1}$ for ${ }^{13} \mathrm{CO}$ and $\mathrm{C}^{18} \mathrm{O}$ ).

in Kowal et al. 2007; Burkhart et al. 2013). Similarly, the integrated intensity map peaks at about $2.5 \mathrm{pc}$, but the channel map shows a peak at a scale of $1.5 \mathrm{pc}\left(10^{\prime}\right.$ corresponds to $\sim 1 \mathrm{pc}$ at a distance of $400 \mathrm{pc}$ ). The $\mathrm{C}^{18} \mathrm{O}$ maps show the same qualitative behavior with somewhat smaller scales, 1.8 and $1.0 \mathrm{pc}$, implied. However, the slope cannot be measured precisely due to higher noise and imaging artifacts. We therefore describe the structure as self-similar at scales up to $0.8 \mathrm{pc}$ and note a relative lack of structure at increasingly larger scales, with only the ISF at the scale of the map.

Applying the same arguments to the channel-map spectra of Figure 4 enables us to determine the maximum size of velocitycoherent structures in our data sets. While on large scales, the contributions of individual filaments can combine to present the appearance of extended, connected structure, our measurements of optically thin lines show that such connections do not always hold in velocity space. The steeper spectrum for our integrated intensity maps is consistent with a positive size-line width relation creating relatively brighter structures at larger scales when integrating over the full velocity profile.

At scales above $2^{\prime}-3^{\prime}$, the ${ }^{12} \mathrm{CO}$ data follow the same trend as ${ }^{13} \mathrm{CO}$, showing a short power-law slope with the same 


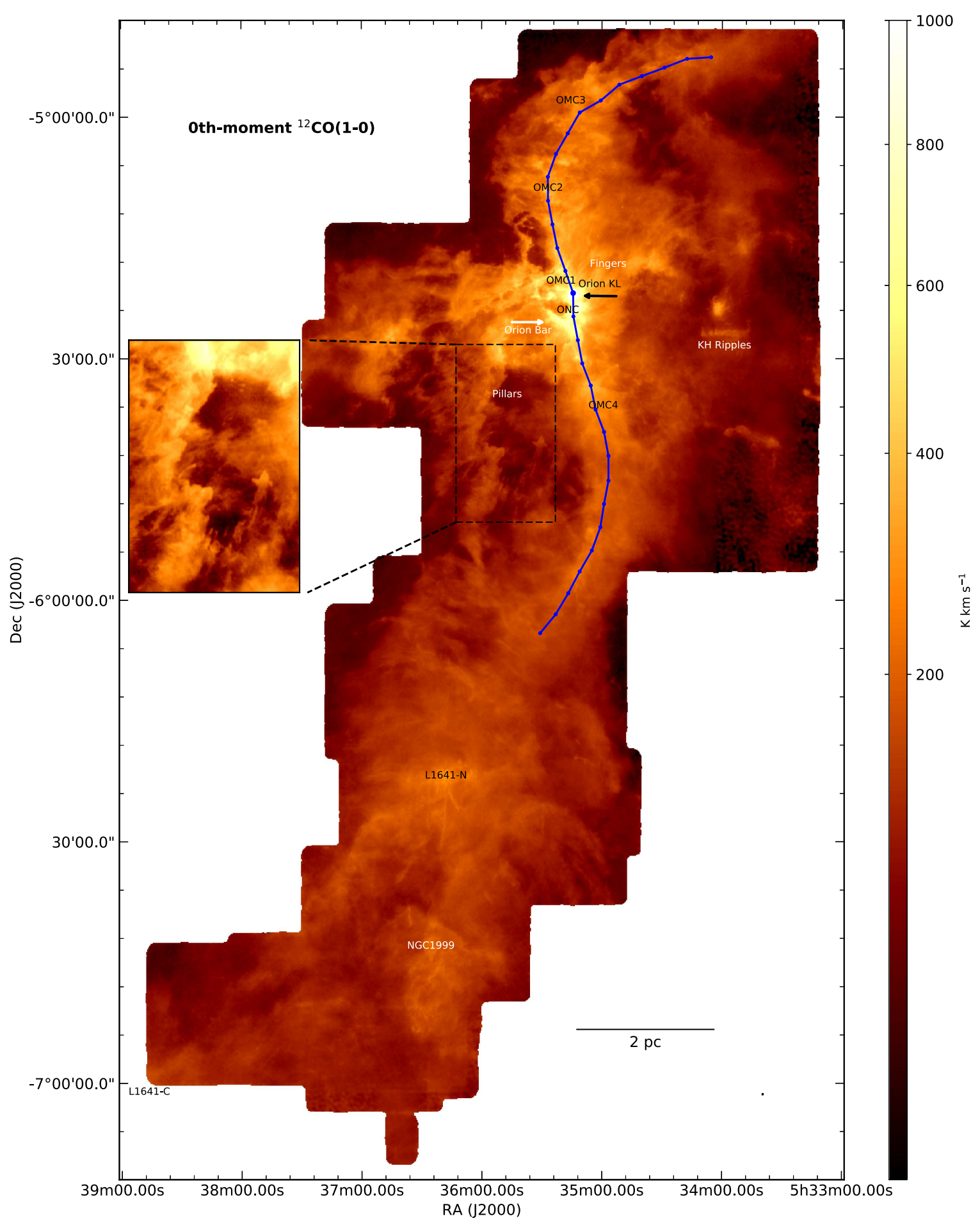

Figure 5. Zeroth-moment (integrated intensity) map of the ${ }^{12} \mathrm{CO}(1-0)$ emission over the velocity range $2.5-15 \mathrm{~km} \mathrm{~s}^{-1}$. No intensity clipping has been applied. The color bar on the right provides the square-root intensity scale in units of $\mathrm{K} \mathrm{km} \mathrm{s}^{-1}$. A zoom-in view of the Pillars is displayed in the inset. Noisier patches seen near R.A. $05^{\mathrm{h}} 33^{\mathrm{m}} 25^{\mathrm{s}} .0$ at various declinations result from limited NRO45 observing time. The blue curve is the cut for the PV diagram defined in Section 4.2 . The blue dots indicate $3^{\prime}$ intervals along the curve.

exponents. However, for ${ }^{12} \mathrm{CO}$, the peaks of the $\Delta$-variance spectra in Figures 3 and 4 are shifted to even larger scales of 2.8 and $2.4 \mathrm{pc}$, respectively, as might be expected in view of the higher optical depth and the sensitivity to somewhat more extended emission. In addition, both maps show a relative excess of structure at a scale of about $1^{\prime}$, corresponding to $0.1 \mathrm{pc}$. This is seen as a prominent bump in the ${ }^{12} \mathrm{CO}$ integrated intensity spectrum. The bump in this optically thick line is most likely attributable to the presence of large velocity gradients and line widths in the contributing regions. The most prominent broad-line region in our maps is Orion KL, followed by some structures south of the Orion Bar. All are approximately $1^{\prime}$ in size. It is worth noting that if a region of a few arcminutes around Orion $\mathrm{KL}$ is masked out, the strength of the bump in the $\Delta$-variance spectra is reduced by $75 \%$. The combination of all $\Delta$-variance spectra is thus consistent with a large-scale 


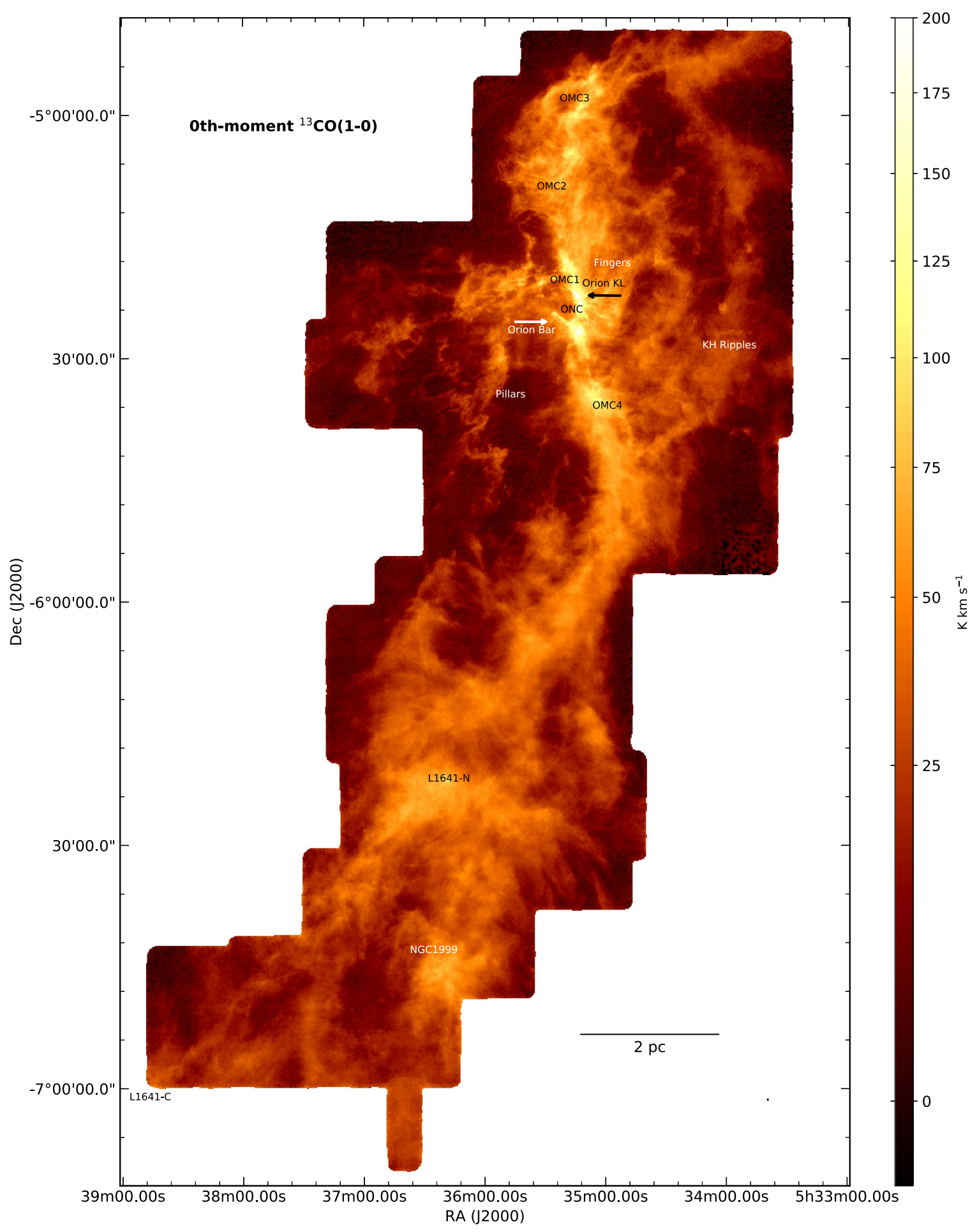

Figure 6. Same as Figure 5 but for ${ }^{13} \mathrm{CO}(1-0)$ emission.

turbulent cascade that is locally modified by dynamical feedback at a scale of about $0.1 \mathrm{pc}\left(1^{\prime}\right)$.

\subsection{Gas Kinematics}

As a first step in understanding the large-scale kinematics of the Orion A cloud, we created false-color images of the integrated intensity maps for ${ }^{12} \mathrm{CO}(1-0)$ and ${ }^{13} \mathrm{CO}(1-0)$ by limiting the integration velocities to three broad ranges-9.8-12.1 km s${ }^{-1}$ (red), 7.3-9.6 km s${ }^{-1}$ (green), and
$4.8-7.1 \mathrm{~km} \mathrm{~s}^{-1}$ (blue) - and combining the resulting maps. These RGB ${ }^{12} \mathrm{CO}(1-0)$ and ${ }^{13} \mathrm{CO}(1-0)$ images are presented in Figures 8 and 9, respectively, and provide broad kinematic information not discernible in the maps in Figures 5 and 6. As before, the intensity scale is in units of $\mathrm{K} \mathrm{km} \mathrm{s}^{-1}$. The most impressive feature in both maps is the large-scale north-south velocity gradient across the length of the cloud. However, it is not clear if the gradient is smooth or merely the result of overlapping clouds along the line of sight. 


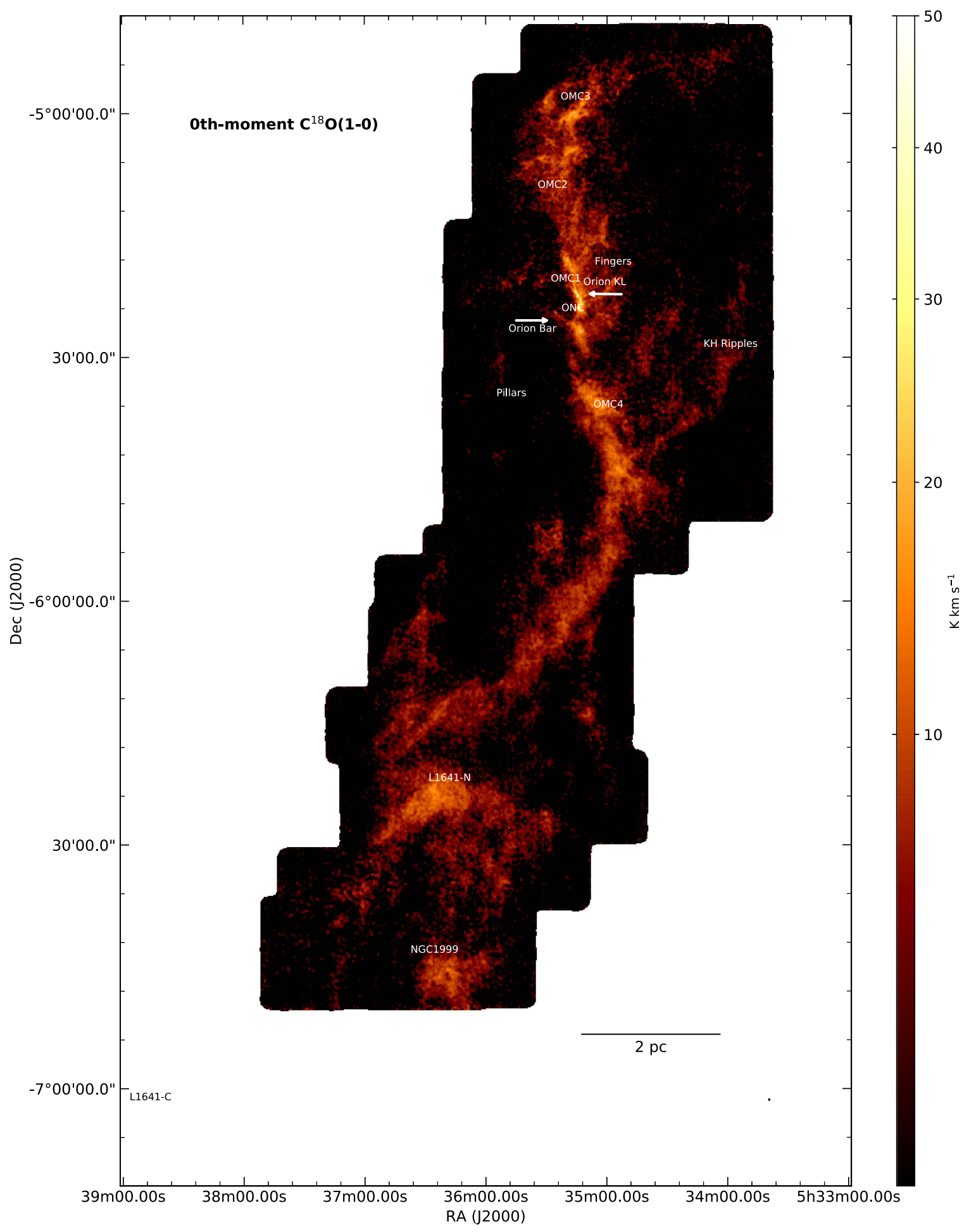

Figure 7. Same as Figure 5 but for $\mathrm{C}^{18} \mathrm{O}(1-0)$ emission.

Due to strong contributions from all three velocity ranges to the ${ }^{12} \mathrm{CO}(1-0)$ emission, the $\sim 6^{\prime}$ radius area around the ONC appears white in Figure 8. This area coincides with the very active OMC-1 region, where a wide range of velocities results from the entrainment of the molecular gas by powerful outflows, especially those associated with the BN/KL objects (Bally et al. 2017). The two areas of anomalously blue emission seen in the otherwise red velocity-dominated region just north of OMC-1 can also be explained in light of previous studies. The northernmost appears to be associated with the northern ionization front (Berné et al. 2014), presumably where the foreground molecular cloud material and the front of the Orion Nebula H II region interact. Green and orange diagonal striations northwest of OMC-1 imply strong feedback effects from the outflow sources in OMC-1.

The strong red emission observed immediately southsoutheast of the ONC is consistent with the long-standing hypothesis that this feature results from the interaction between 


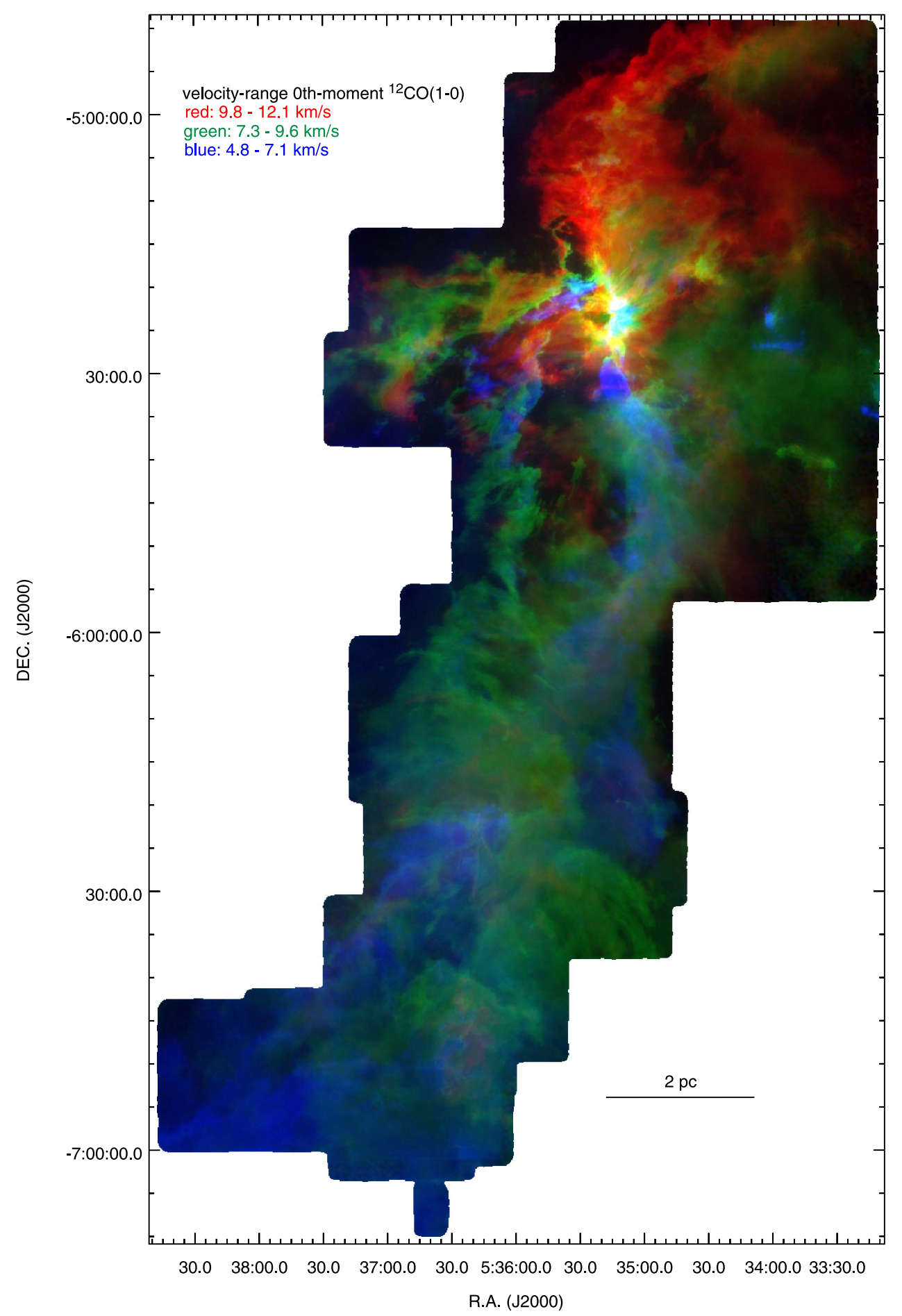

Figure 8. RGB false-color image of velocity-limited integrated intensity maps for ${ }^{12} \mathrm{CO}(1-0)$. Integration ranges and colors are $4.8-7.1 \mathrm{~km} \mathrm{~s}^{-1}$ (blue), 7.3-9.6 km s ${ }^{-1}$ (green), and $9.8-12.1 \mathrm{~km} \mathrm{~s}^{-1}$ (red).

the far side of the expanding Orion Nebula $\mathrm{H}$ II region and the molecular cloud (Loren 1979; Berné et al. 2014). The irradiated pillar-like globules noted in Figure 5 to the south and southeast of the ONC appear as green and blue velocity features in Figures 8 and 9. They are thought to be primarily in the "dark lane south filament" on the near side of the cloud (RodriguezFranco et al. 1998; Shimajiri et al. 2011, 2013). Similar mixtures of blue and green emission are seen in the L1641 cloud and the southern part of the ISF. Nakamura et al. (2012) proposed that a cloud-cloud collision in the area triggered the formation of the filaments and initiated SF in the L1641-N cluster.

Velocity channel maps from our combined data cubes reveal the complex kinematics of Orion $\mathrm{A}$ in considerable detail. Figures 10-12 display sample channel maps for ${ }^{12} \mathrm{CO}(1-0)$, ${ }^{13} \mathrm{CO}(1-0)$, and $\mathrm{C}^{18} \mathrm{O}(1-0)$, respectively. Movies showing the channel maps over the full bandwidth for each cube are linked to the online versions of these figures. Complete channel maps for all three cubes at full velocity resolution are presented in the 


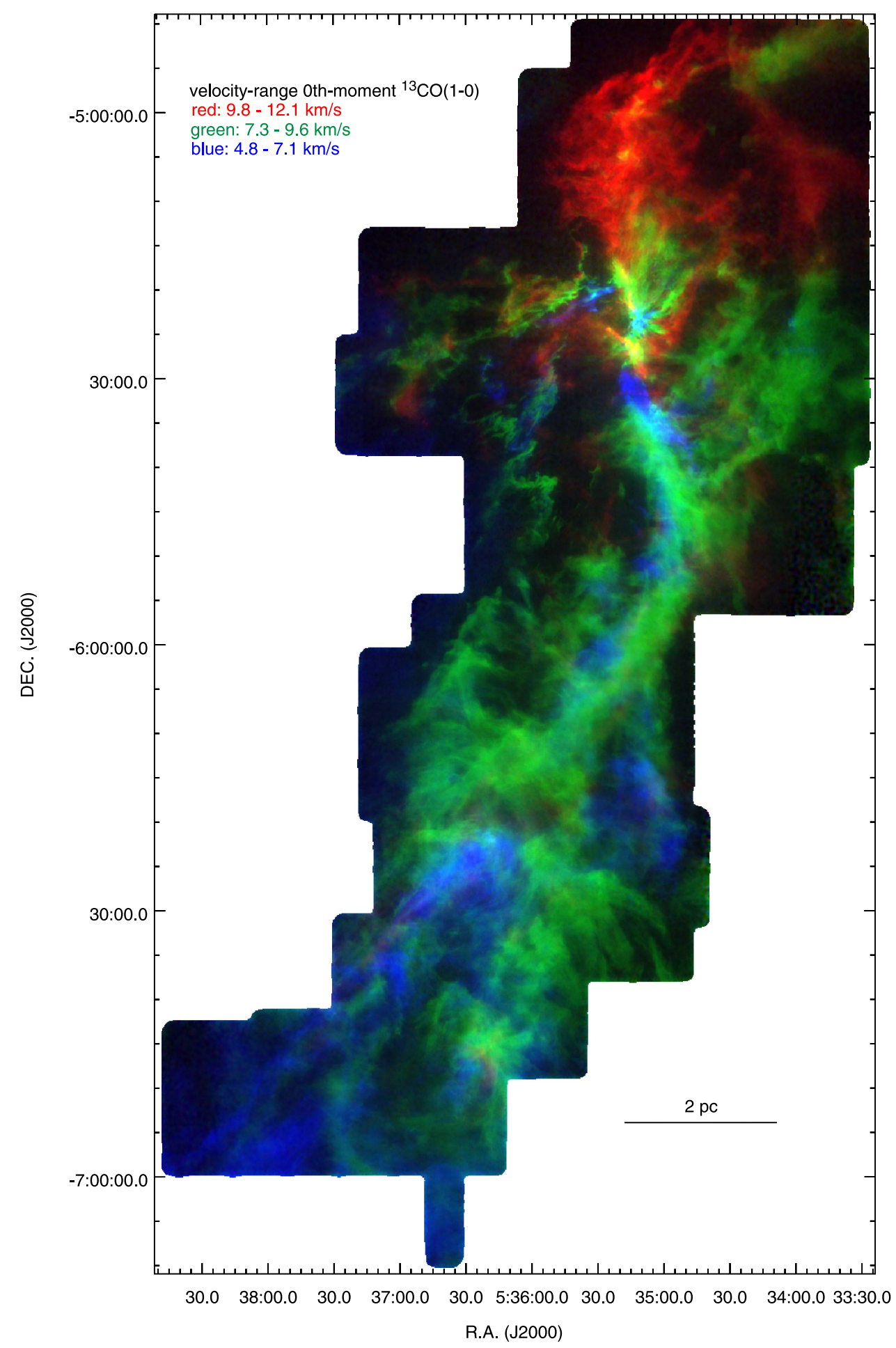

Figure 9. Same as Figure 8 but for ${ }^{13} \mathrm{CO}(1-0)$. The three integration ranges and colors are 4.8-7.1 $\mathrm{km} \mathrm{s}^{-1}$ (blue), 7.3-9.6 km s${ }^{-1}$ (green), and 9.8-12.1 km s${ }^{-1}$ (red).

Appendix (Figures 26-28). Features clearly visible in these maps include the clumpy structure at various velocities in the L1641 region, the high-velocity gas from the Orion $\mathrm{KL}$ (OMC-1) explosive outflow, and various filaments in the OMC-2/3 region.

The north-south velocity shift seen in the RGB maps of Figures 8 and 9 can also be traced by considering the molecular-line emission at various positions across the Orion A cloud. Figure 13 shows the averaged spectral-line profiles for ${ }^{12} \mathrm{CO}(1-0),{ }^{13} \mathrm{CO}(1-0)$, and $\mathrm{C}^{18} \mathrm{O}(1-0)$ for the four regions of the cloud defined in Figure 1. As expected, the velocity of the peak intensity for each $\mathrm{CO}$ species shifts from $\sim 11$ to $7.5 \mathrm{~km} \mathrm{~s}^{-1}$, moving from north to south in the cloud, consistent with the large-scale change in velocity seen in the RGB maps. The double-peaked lines seen in the L1641 "region" have been noted previously and gave rise to the cloud-cloud collision scenario suggested by Nakamura et al. (2012).

The overall north-to-south shift in velocity can also be seen in our first-moment maps for ${ }^{12} \mathrm{CO}(1-0),{ }^{13} \mathrm{CO}(1-0)$, and 


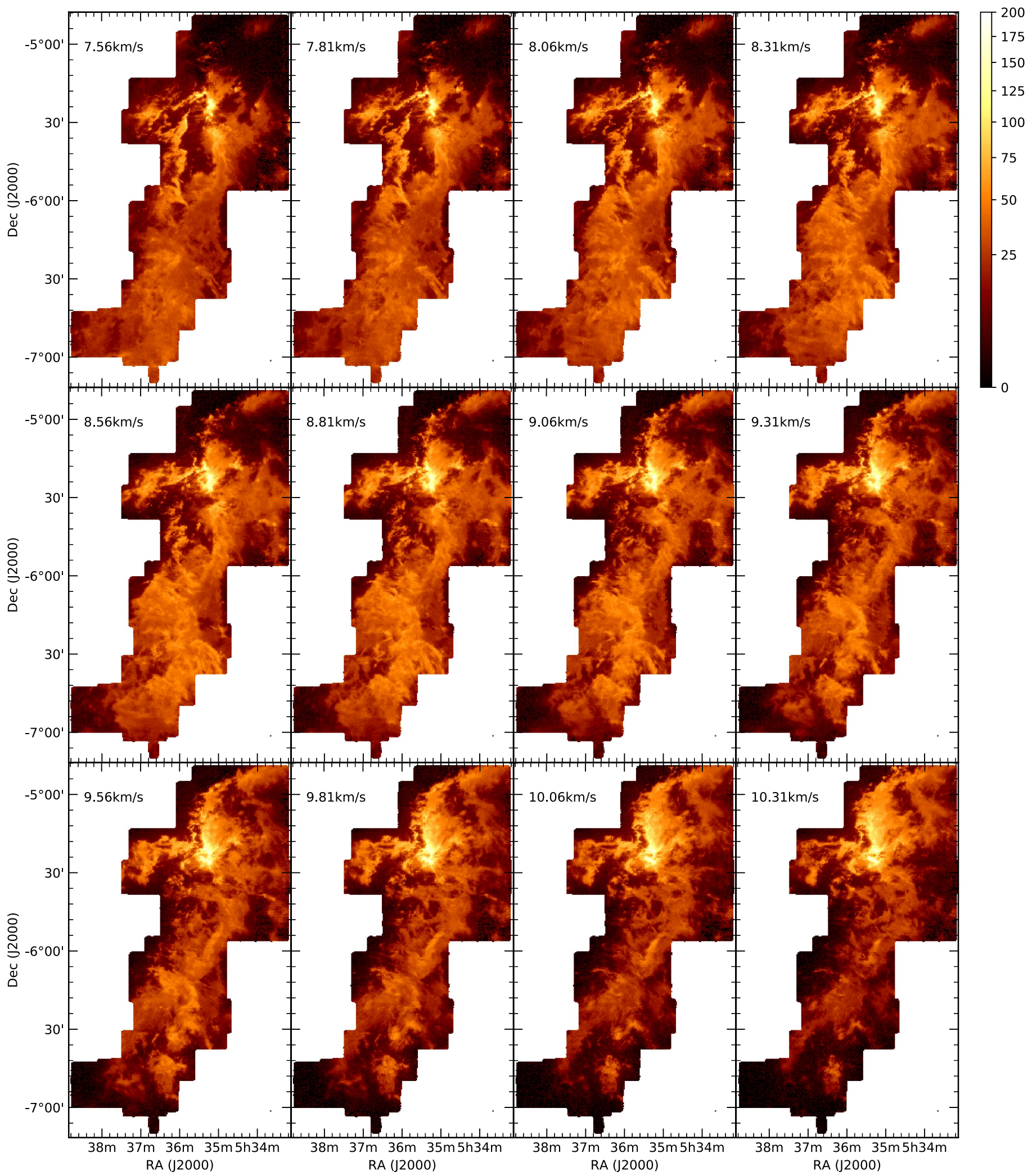

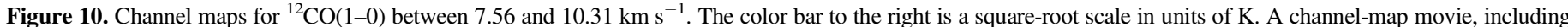
all channels, is available. The animation starts at $-1.9 \mathrm{~km} \mathrm{~s}^{-1}$ and ends at $19.1 \mathrm{~km} \mathrm{~s}^{-1}$. The duration is $17 \mathrm{~s}$. Figure 26 , in the Appendix, shows all channel maps.

(An animation of this figure is available.)

$\mathrm{C}^{18} \mathrm{O}(1-0)$ (Figures 14-16, respectively). Again, the northern part of the cloud is preferentially redder, indicating higher velocities, while the southern part is, for the most part, blue and at lower velocities. The origin of the gradient has been discussed at length in the literature but is not yet fully understood. To date, explanations put forward include rotation, filament expansion, and global collapse (Allen \& Davis 2008 and references therein). The high-resolution, high-sensitivity 


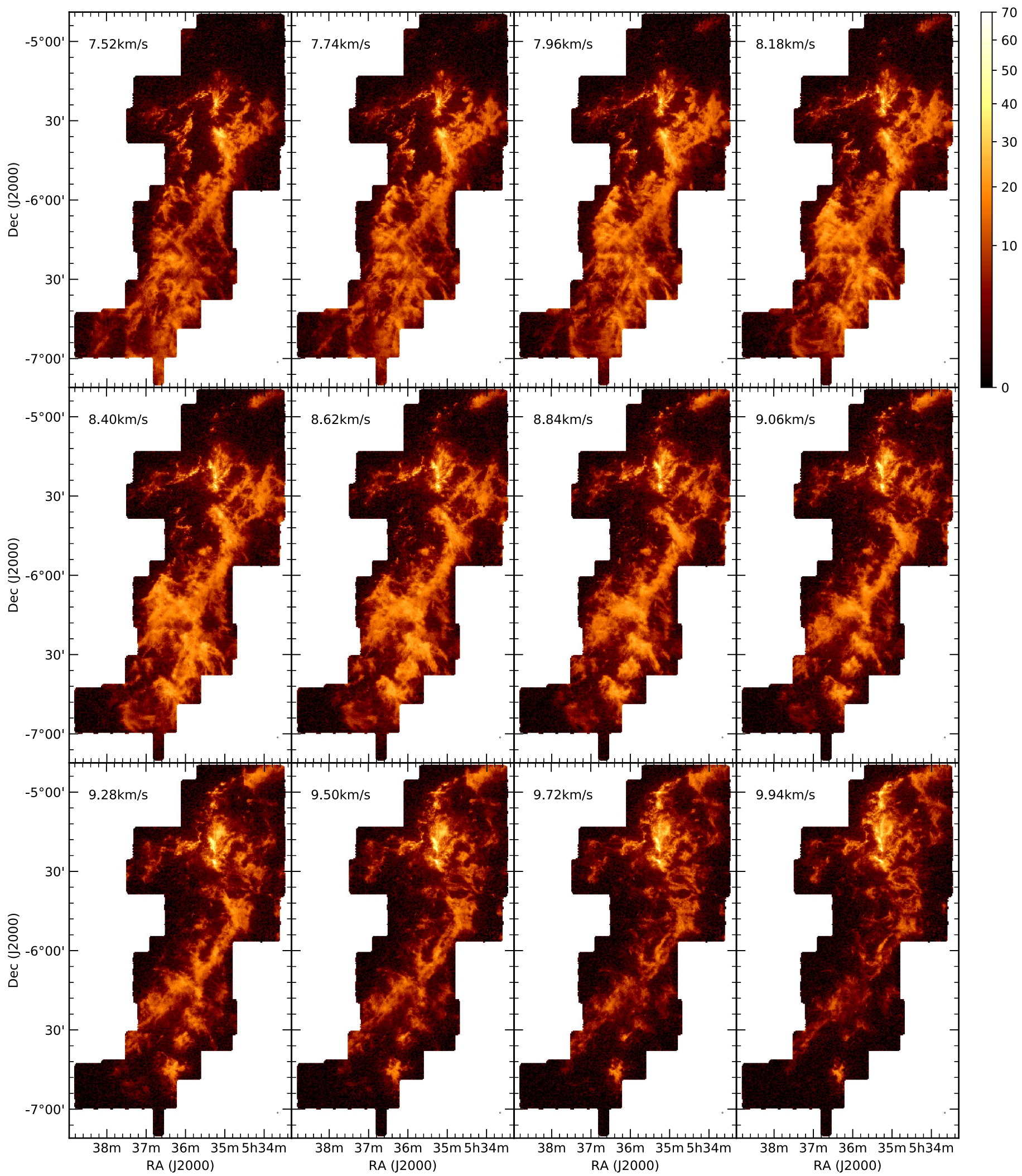

Figure 11. Same as Figure 10 but for the ${ }^{13} \mathrm{CO}(1-0)$ emission. A channel-map movie, including all channels, is available. The animation starts at $0.3 \mathrm{~km} \mathrm{~s}{ }^{-1}$ and ends at $16.4 \mathrm{~km} \mathrm{~s}^{-1}$. The duration is $30 \mathrm{~s}$. Figure 27, in the Appendix, shows all channel maps.

(An animation of this figure is available.)

CARMA-NRO Orion data set presented here offers a new opportunity to test every hypothesis in detail over the requisite extended scales.
In addition, these first-moment maps present, in much more detail, the variation in velocity with position in the cloud over the entire velocity integration range, $2.5-15 \mathrm{~km} \mathrm{~s}^{-1}$. Like the 


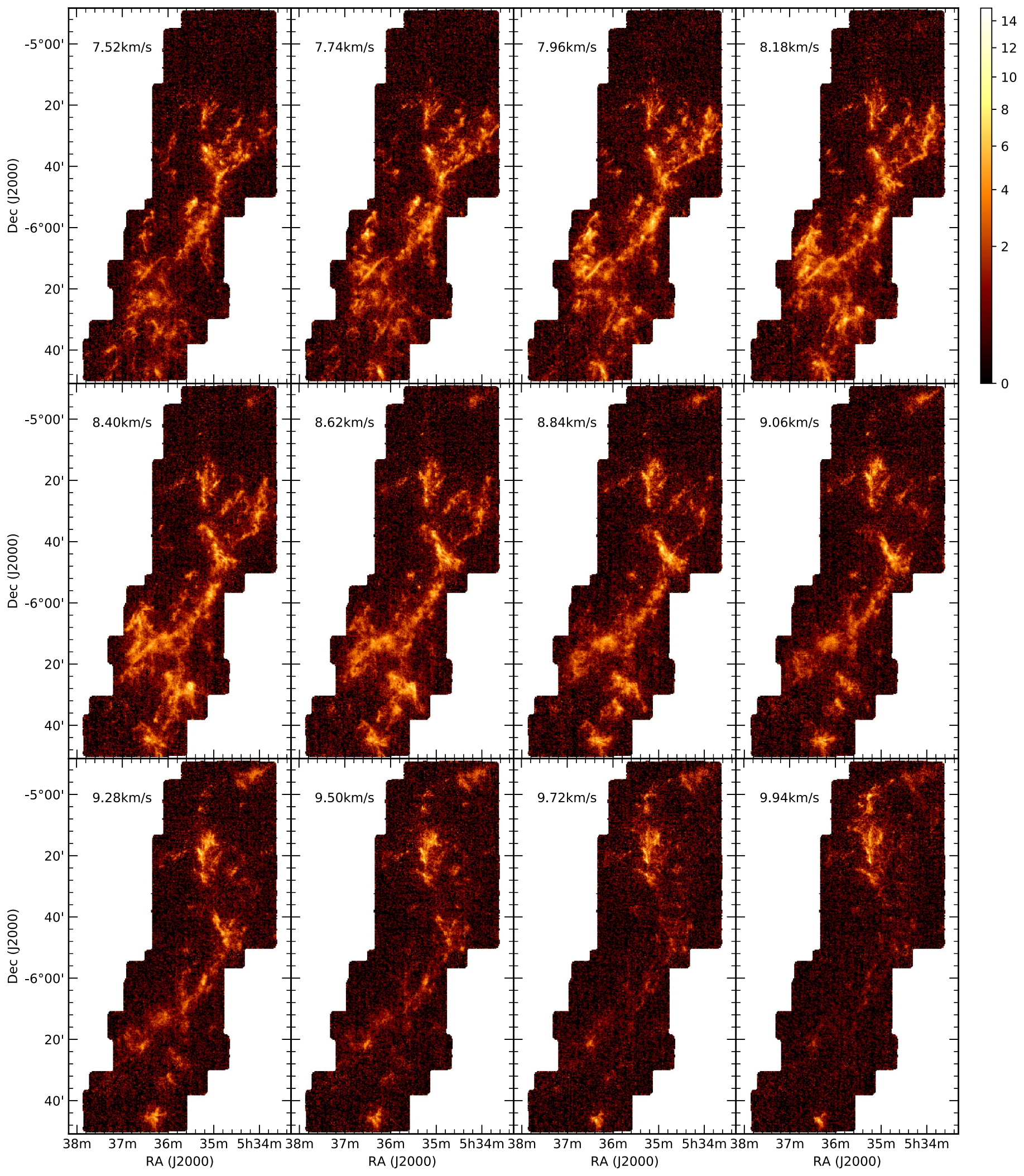

Figure 12. Same as Figure 10 but for the $\mathrm{C}^{18} \mathrm{O}(1-0)$ emission. A channel-map movie, including all channels, is available. The animation starts at $0.3 \mathrm{~km} \mathrm{~s}{ }^{-1}$ and ends at $16.5 \mathrm{~km} \mathrm{~s}^{-1}$. The duration is $30 \mathrm{~s}$. Figure 28, in the Appendix, shows all channel maps.

(An animation of this figure is available.)

maps of gas distribution, they show interesting features, often noted by earlier focused studies, in a larger-scale context. Among these is a distinctive elongated blue field to the west of OMC-1 in Figure 14. This coincides with the location of the
Kelvin-Helmholtz "ripples" that are thought to be in the foreground of the ONC (Berné et al. 2010). Likewise, in Figure 16, there appears to be a velocity gradient along the short axis of the filament below OMC-4, similar to that seen in 

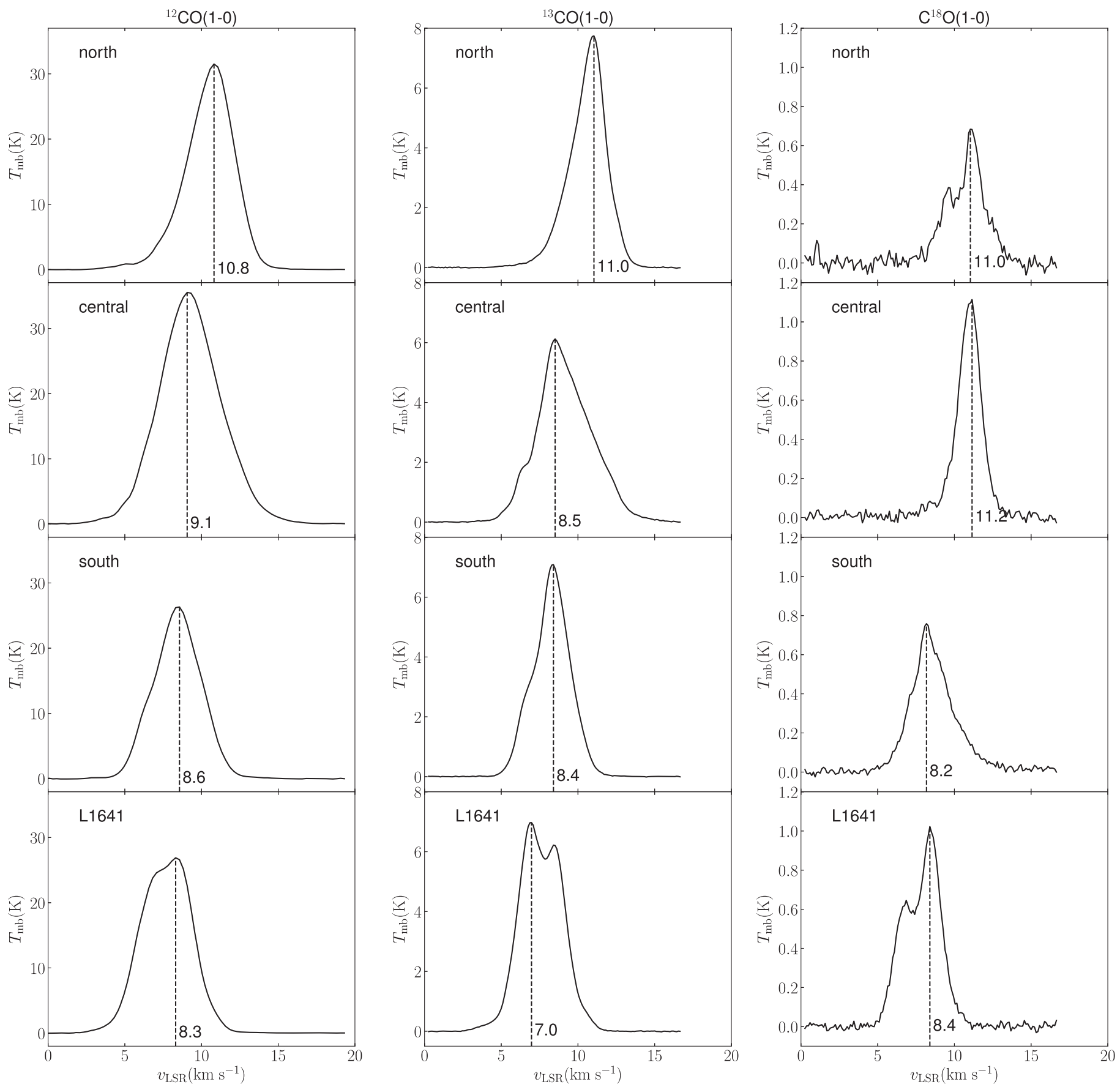

Figure 13. Averaged profiles for ${ }^{12} \mathrm{CO}(1-0)$ (left), ${ }^{13} \mathrm{CO}(1-0)$ (middle), and $\mathrm{C}^{18} \mathrm{O}(1-0)$ (right) in the four areas of the Orion A cloud shown in Figure 1. The line profiles were constructed for each separate region by averaging the intensities in each velocity channel. Different intensity scales are required for each molecular species, but the velocity scale is the same for all. Vertical dashed lines mark the velocity of peak emission in each case.

simulations of $\mathrm{C}^{18} \mathrm{O}(1-0)$ emission from a filament in a turbulent cloud by Smith et al. (2016). These authors attributed the gradient to the filament moving across the cloud as a coherent velocity front, corresponding to one of the large-scale modes in a turbulent cascade. Given the results of our $\Delta$-variance analysis, it is tempting to suggest that a similar mechanism may be at work here. More importantly, this highlights yet again the potential impact of our new survey on the understanding of large-scale processes in molecular clouds.

Interesting features in Figure 14 include several prominent bipolar outflows. They are noticeable because the velocities of the outflowing molecular gas differ significantly from the systemic cloud velocity in their neighborhoods, increasing the contrast with the cloud. The effect is most easily seen in the Figure 14 insets. In the southern part of the cloud, elongated deep blue outflow lobes contrast with cyan/light green regions. In the north, extended red/yellow lobes contrast with light green structures. Identified examples of bipolar outflows in the map include V380 Ori-N (Davis et al. 2000), L1641-N (Stanke \& Williams 2007), and OMC-2/3 MMS 9 (e.g., Takahashi et al. 2008). There are certainly many more outflows in the region (see, e.g., Williams et al. 2003; Takahashi et al. 2008; Nakamura et al. 2012). However, if these are low-intensity emission with velocities similar to the systemic value or are 


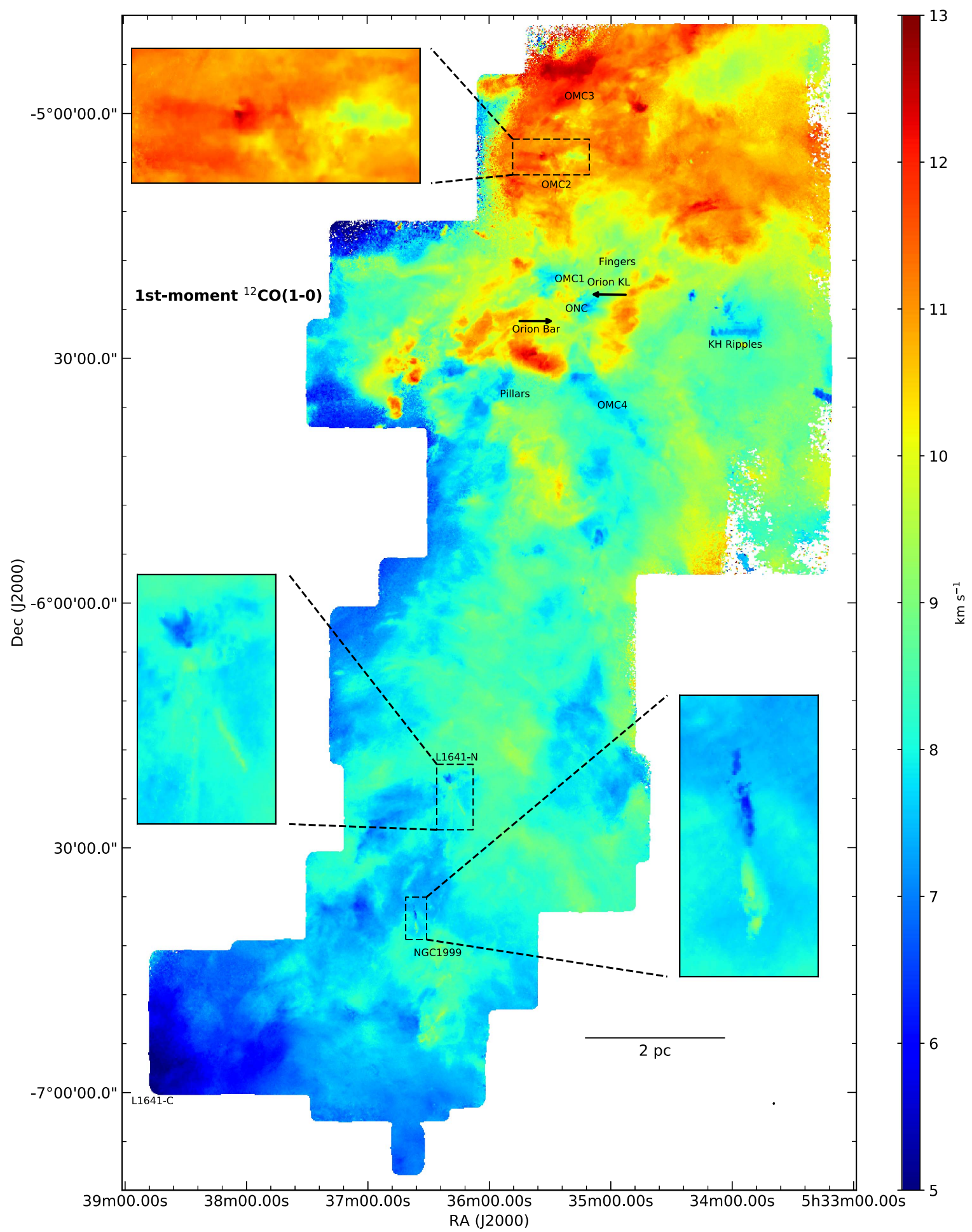

Figure 14. First-moment map showing the variation of peak ${ }^{12} \mathrm{CO}(1-0)$ velocities between 2.5 and $15 \mathrm{~km} \mathrm{~s}^{-1}$ as a function of position in the Orion A cloud. Only emission above $5 \sigma$ is used to produce the map. The color bar to the right is a linear scale in units of $\mathrm{km} \mathrm{s}^{-1}$. The insets show zoom-in views of three interesting outflows.

small or unipolar, they will be difficult to detect in the firstmoment map.

Signposts of feedback can also be detected in maps that display the variation of velocity dispersion across the Orion A cloud. These are provided in the second-moment maps of ${ }^{12} \mathrm{CO},{ }^{13} \mathrm{CO}$, and $\mathrm{C}^{18} \mathrm{O}$ in Figures $17-19$, respectively. For the most part, all three maps show a smooth distribution, with dispersions mostly between 0.5 and $1.5 \mathrm{~km} \mathrm{~s}^{-1}$ but occasionally reaching $2 \mathrm{~km} \mathrm{~s}^{-1}$. The highest values coincide with the Orion KL region and are likely attributable to the highvelocity explosive outflow (Bally et al. 2017). Significantly high-velocity dispersions are also noticeable in the region around the ONC characterized as the "shell around Orion-KL" (Shimajiri et al. 2011) and may be due to feedback effects from the ONC region and overlapping line-of-sight velocity components. In Figure 17, high-velocity dispersions are also 


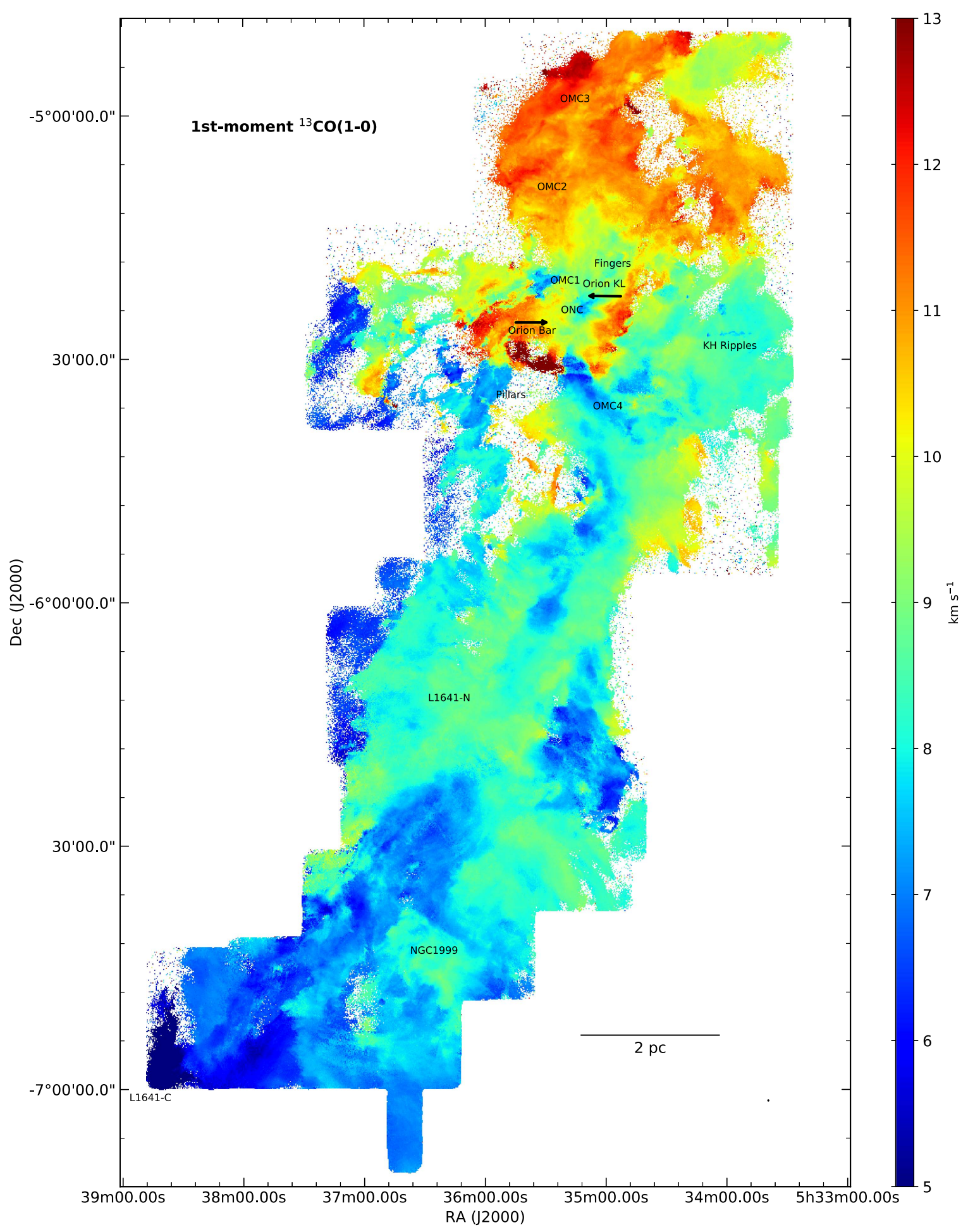

Figure 15. Same as Figure 14 but for the ${ }^{13} \mathrm{CO}(1-0)$ emission.

associated with molecular outflows in NGC 1999, L1641-N, and $\mathrm{OMC}-2 / 3$.

Another way to examine how gas kinematics change with location in the cloud relies on position-velocity (PV) diagrams for the regions of interest. As an example, in Figures 20-22, we show the ${ }^{12} \mathrm{CO},{ }^{13} \mathrm{CO}$, and $\mathrm{C}^{18} \mathrm{O}$ PV diagrams along the ISF. The blue curve in Figure 5 defines the PV cut, and a large blue dot at the position of Orion KL represents the reference position (R.A.J2000 $=5^{\mathrm{h}} 35^{\mathrm{m}} 13^{\mathrm{s}} .0, \quad$ decl. $_{\mathrm{J} 2000}=-5^{\circ} 22^{\prime} 05^{\prime \prime}$ 0). Positive offsets are toward the north. We extract a beam-averaged spectrum every $3^{\prime \prime}$ along the PV cut. The velocity resolutions in the PV diagrams for each cube are as given in Table 2.

The broad velocity spread at the reference position is prominent in all three PV diagrams, especially in Figure 20, and reflects the high velocities of the explosive outflow known to be present in the Orion KL region (Bally et al. 2017). Other high-velocity spikes can be discerned in Figure 20, at offsets $16^{\prime}, 12^{\prime}$, and $-37^{\prime}$, for example, and are likely due to bipolar 


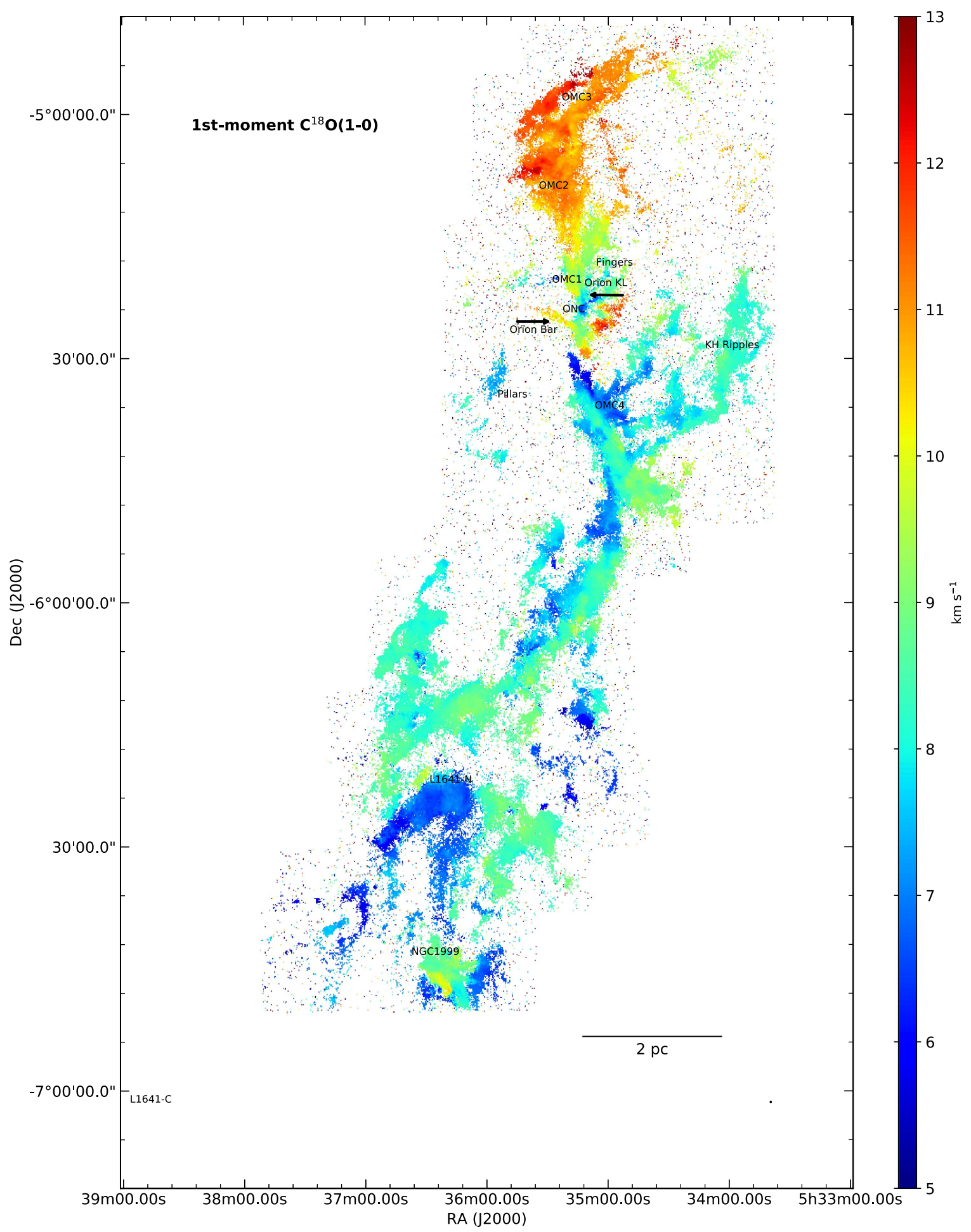

Figure 16. Same as Figure 14 but for the $\mathrm{C}^{18} \mathrm{O}(1-0)$ emission.

outflows. A bubble-like structure at offset $-10^{\prime}$ in Figures 21 and 22 is probably associated with the "shell around OrionKL" mentioned earlier. The overall sinusoidal shape present in our PV diagrams is very intriguing. Stutz \& Gould (2016) and Schleicher \& Stutz (2018) proposed the slingshot model, in which the gas is oscillating and ejecting protostars in an autodestructive cycle that ultimately results in cluster formation. The observed morphology in our PV diagrams is strongly reminiscent of such a wavelike motion. However, the exact nature of this wave remains unclear. Our observed signature is inconsistent with a propagating wave because this would require that the maximum displacement corresponds to the minimum velocity, in contradiction to Figures 20-22. These figures show that the maximum displacement coincides with the position of maximum velocity, most clearly seen in the OMC-2/3 regions, located near offset $20^{\prime}$. Waves of other types (such as standing or torsional) may better explain the data. Further analysis is needed for the types of instabilities that 


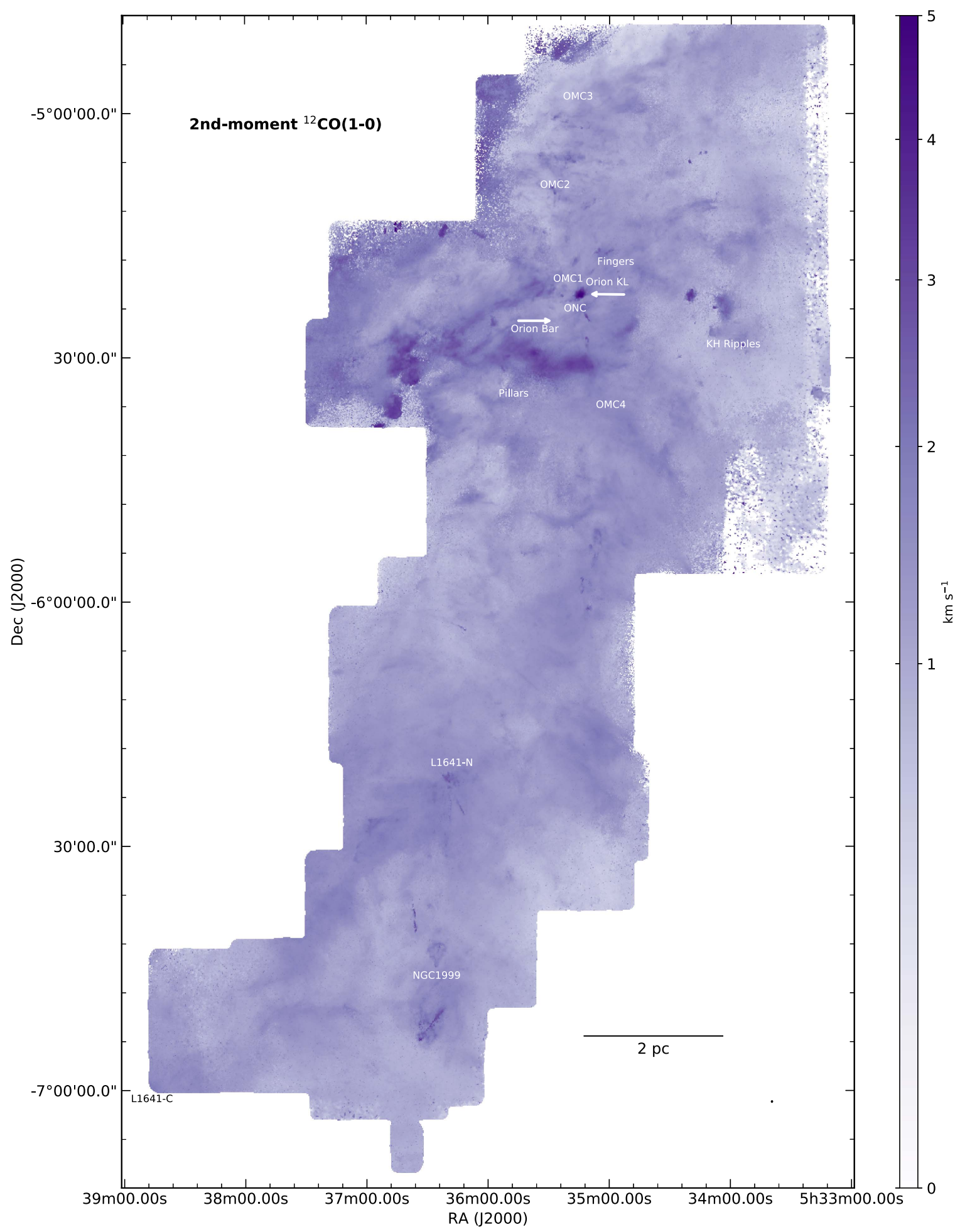

Figure 17. Second-moment map of the ${ }^{12} \mathrm{CO}(1-0)$ emission over the velocity range $2.5-15 \mathrm{~km} \mathrm{~s}^{-1}$, showing the variation of the velocity dispersion of ${ }^{12} \mathrm{CO}$. Only emission above $5 \sigma$ is used to produce the map. The color bar has a square-root scale with units of $\mathrm{km} \mathrm{s}^{-1}$.

may give rise to our observed PV diagram morphology (e.g., Schleicher \& Stutz 2018). This is one example where the data presented here could be use to constrain models of different kinds of processes in molecular clouds and underlines the vast reservoir of kinematic information that becomes available with the CARMA-NRO survey. Overall, the results provide an unprecedented opportunity for increasing our understanding of energetic phenomena in star-forming clouds.

\section{Discussion}

\subsection{Gas Excitation Temperature}

Measurements of the excitation temperature and column density of the molecular gas across the Orion A cloud are critical to interpreting the results presented thus far. Figure 23 shows the variation of excitation temperature, $T_{\mathrm{ex}}$, across the mapped area of the Orion cloud. Values of $T_{\mathrm{ex}}$ were determined 


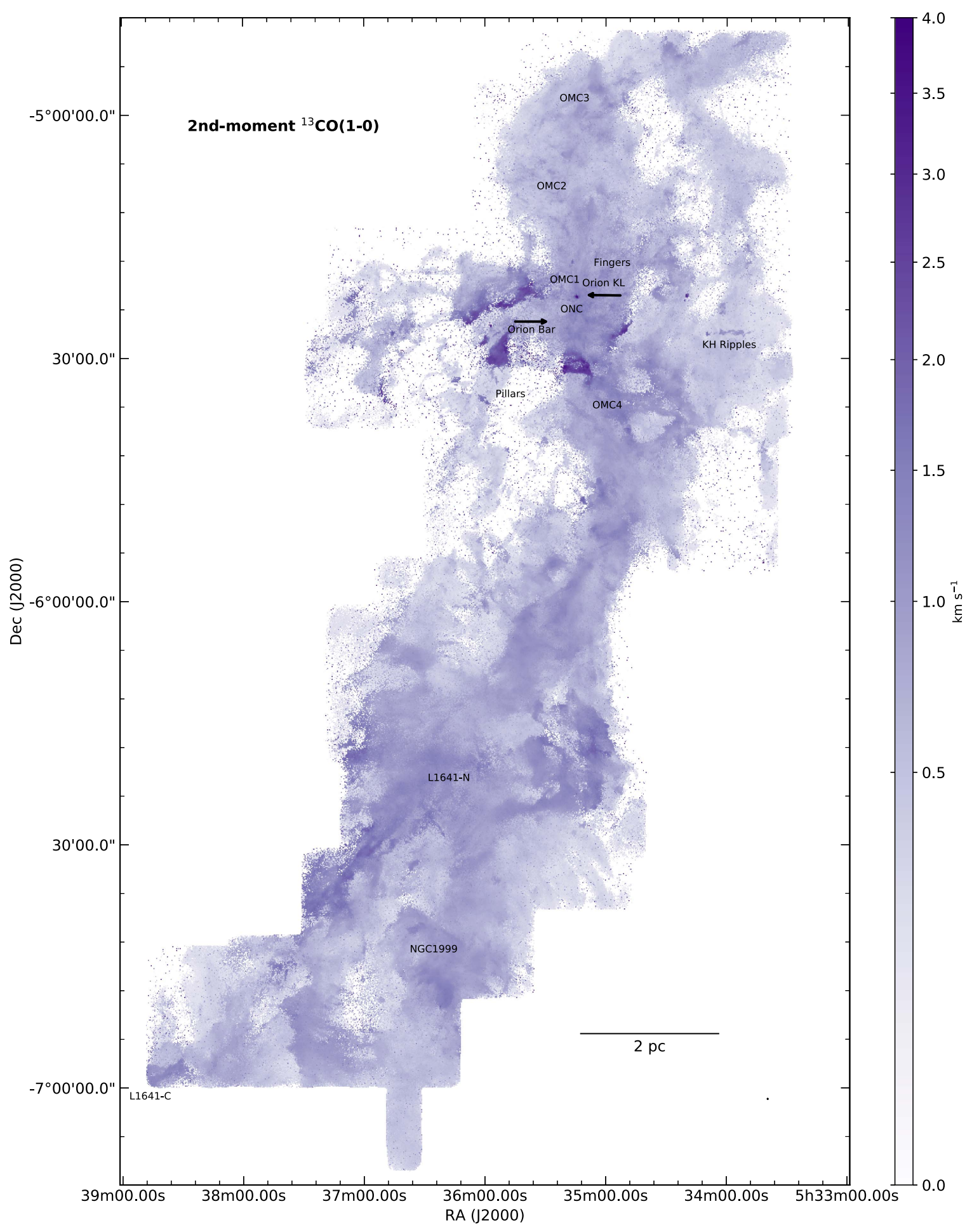

Figure 18. Same as Figure 17 but for ${ }^{13} \mathrm{CO}(1-0)$.

from the peak intensities of the ${ }^{12} \mathrm{CO}(1-0)$ line in velocity space, $T_{\max }\left({ }^{12} \mathrm{CO}\right)$, assuming the line is optically thick. Following Pineda et al. (2008), we use

$$
T_{\text {ex }}=\frac{5.5 \mathrm{~K}}{\ln \left\{1+5.5 \mathrm{~K} /\left[T_{\max }\left({ }^{12} \mathrm{CO}\right)+0.82 \mathrm{~K}\right]\right\}} .
$$

The maximum and minimum derived values for $T_{\mathrm{ex}}$ in the map are 220 and $2.8 \mathrm{~K}$, respectively, with a mean of $43.6 \mathrm{~K}$ and a median value of $41.2 \mathrm{~K}$. As a check, we compared our derived excitation temperatures with those derived from the IRAM $30 \mathrm{~m}{ }^{12} \mathrm{CO}(2-1)$ map by Berné et al. (2014) that covers the region from OMC-3 in the north to just south of OMC-4. The combined CARMA+NRO45 map was smoothed to the $\sim 11^{\prime \prime}$ resolution of the IRAM $30 \mathrm{~m}$ map, and the $T_{\text {ex }}$ was estimated from the peak intensity of the line at each position. Within the inner quarter of the IRAM $30 \mathrm{~m}$ map, with the best signal-to-noise ratio, most values coincide within $35 \%$. 


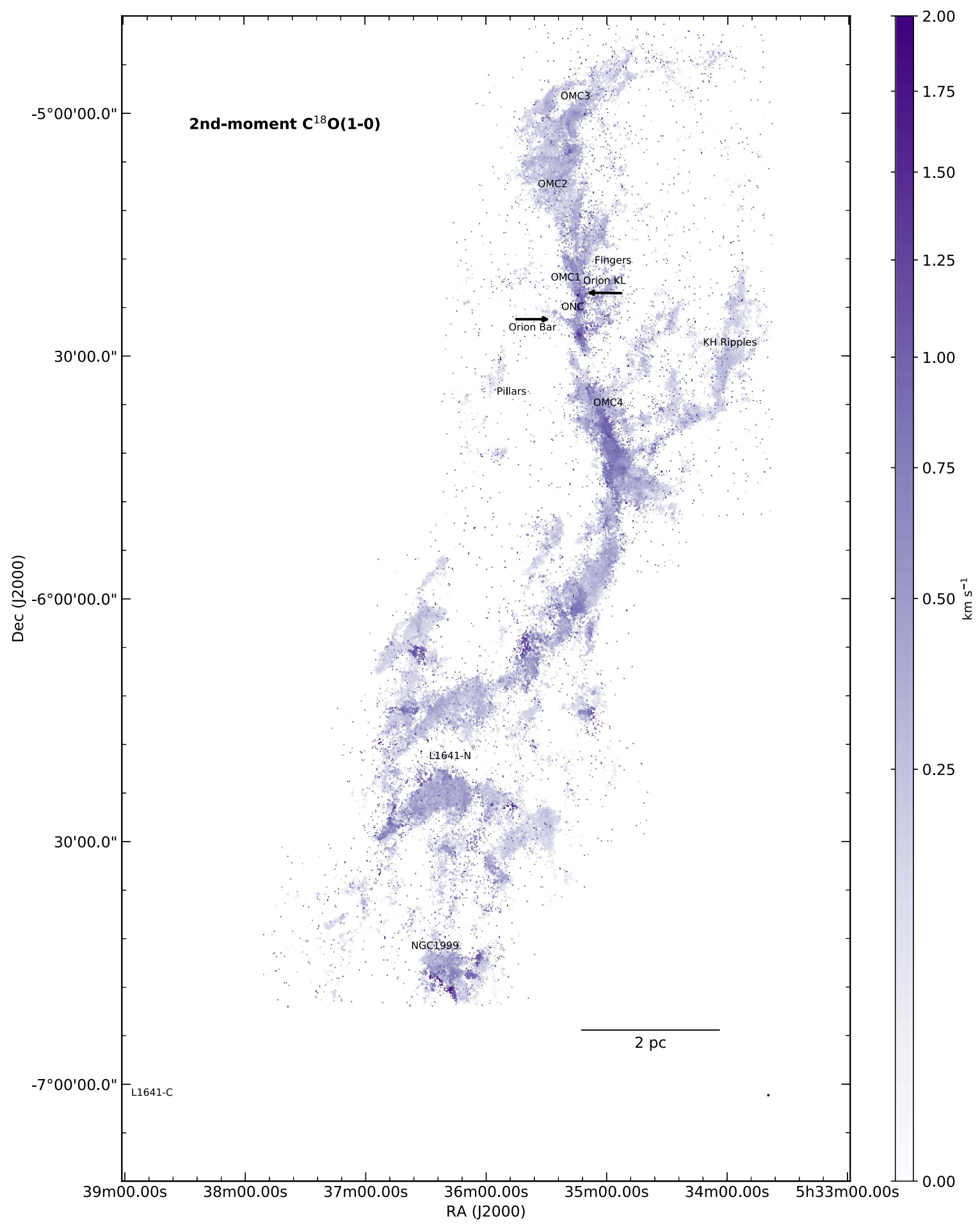

Figure 19. Same as Figure 17 but for $\mathrm{C}^{18} \mathrm{O}(1-0)$.

The excitation temperature distribution in Figure 23 shows a number of trends. At the northern boundary of the map-in effect, the northern edge of OMC-3 - temperatures range from 80 to $110 \mathrm{~K}$. It is likely that these relatively high temperatures result from the interaction of ionizing photons from the NGC 1973/1975/1977 H II region beyond the map boundary. Moving south toward OMC-2 along the ISF, temperatures first decrease $\left(T_{\mathrm{ex}} \sim 60 \mathrm{~K}\right)$ but rise again to the west of the M43 H II region. The highest temperatures are in the OMC-1/Bar region and are usually around $170 \mathrm{~K}$ but can reach $220 \mathrm{~K}$. These values are consistent with estimates of the gas kinetic temperature in the OMC-1 and Bar region derived from para- $\mathrm{H}_{2} \mathrm{CO}$ line ratios (Tang et al. 2018) and excitation temperatures derived from observations of $\mathrm{CO}(6-5)$ (Peng et al. 2012) and $\mathrm{CO}(9-8)$ (Kawamura et al. 2002).

Southeast of the ONC, in the Pillars region, $T_{\mathrm{ex}}$ always increases toward the tips of these structures closer to the ONC, consistent with heating by the UV radiation from its high-mass stars. South of the ONC, temperatures continue to decrease along the ISF as the distance from the new high-mass stars 


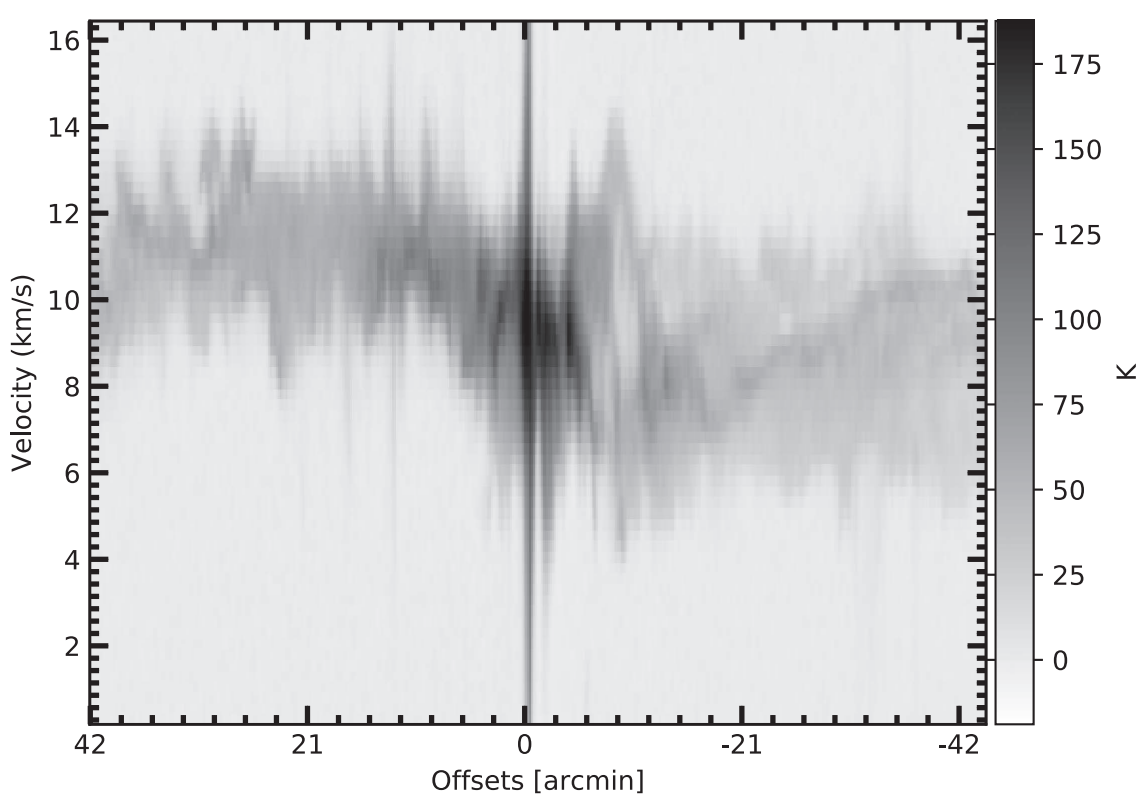

Figure 20. The PV diagram of the ${ }^{12} \mathrm{CO}(1-0)$ emission along the ISF (see Figure 5). The reference (offset $=0$ ) position coincides with the position of Orion KL, with positive offsets to the north. The grayscale bar is linear and in units of main-beam temperature (K).

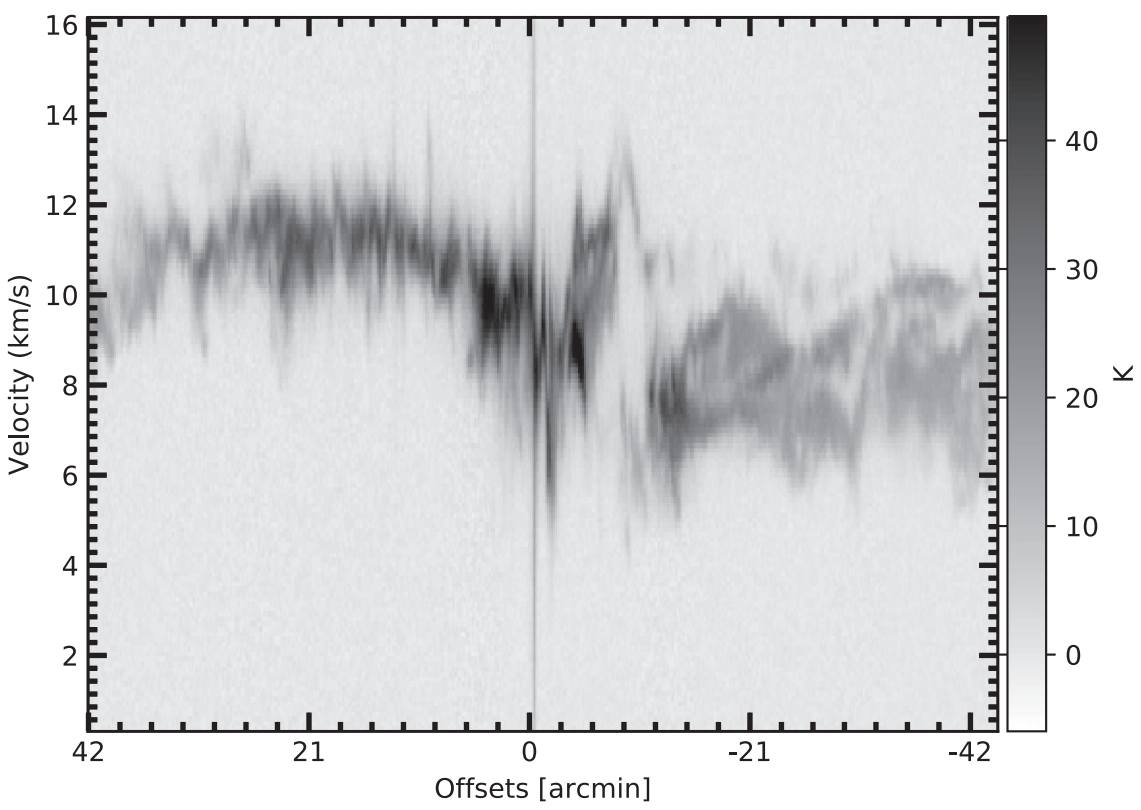

Figure 21. Same as Figure 20 but for ${ }^{13} \mathrm{CO}(1-0)$.

increases, reaching about $50 \mathrm{~K}$ at the southern edge. Further south, average temperatures remain relatively stable. For example, in L1641, values of $30-40 \mathrm{~K}$ are seen in the eastern region and $40-50 \mathrm{~K}$ in the west. These are relatively high temperatures compared to low-mass star-forming regions such as Perseus and Taurus, where $T_{\mathrm{ex}} \sim 10-20 \mathrm{~K}$ (Pineda et al. 2008, 2010). The enhancement is probably the result of $\mathrm{CO}$ tracing the warm surface of the cloud that is heated by the many relatively nearby $\mathrm{OB}$ stars.

\subsection{Gas Column Density}

The column density of gas, $N_{13}$, was determined from our ${ }^{13} \mathrm{CO}(1-0)$ data, assuming the excitation temperatures derived for ${ }^{12} \mathrm{CO}(1-0)$. Following Pineda et al. (2008), ${ }^{13} \mathrm{CO}$ optical depths, $\tau_{13}$, were calculated at each pixel. The ${ }^{13} \mathrm{CO}$ column densities, $N_{13}$, were then calculated as described in Bourke et al. (1997) for those pixels where the signal-to-noise ratio exceeded 3 . If the regions probed by ${ }^{13} \mathrm{CO}(1-0)$ emission are cooler than the surface areas probed by ${ }^{12} \mathrm{CO}$, the absolute column densities of ${ }^{13} \mathrm{CO}$ will be underestimated. However, the overall variations across the map will be relatively unaffected. For most pixels, the emission is optically thin. Only about $0.6 \%$ of the pixels have $\tau_{13}>1$, and these are mostly in the OMC-2/3 and L1641-N regions.

The resulting column density map is shown in Figure 24 . Values of $N_{13}$ range from $2.1 \times 10^{14}$ to $3.2 \times 10^{18} \mathrm{~cm}^{-2}$. The mean is $6.5 \times 10^{16} \mathrm{~cm}^{-2}$, and the median is $3.9 \times 10^{16} \mathrm{~cm}^{-2}$. The highest values of $N_{13}$ are seen in the OMC-1 and Bar regions, with values of approximately $(0.8-3) \times 10^{18} \mathrm{~cm}^{-2}$. Relatively high values of the column density, $(2-5) \times 10^{17} \mathrm{~cm}^{-2}$, are also evident in the OMC-2 and OMC- 3 regions of active SF, and 


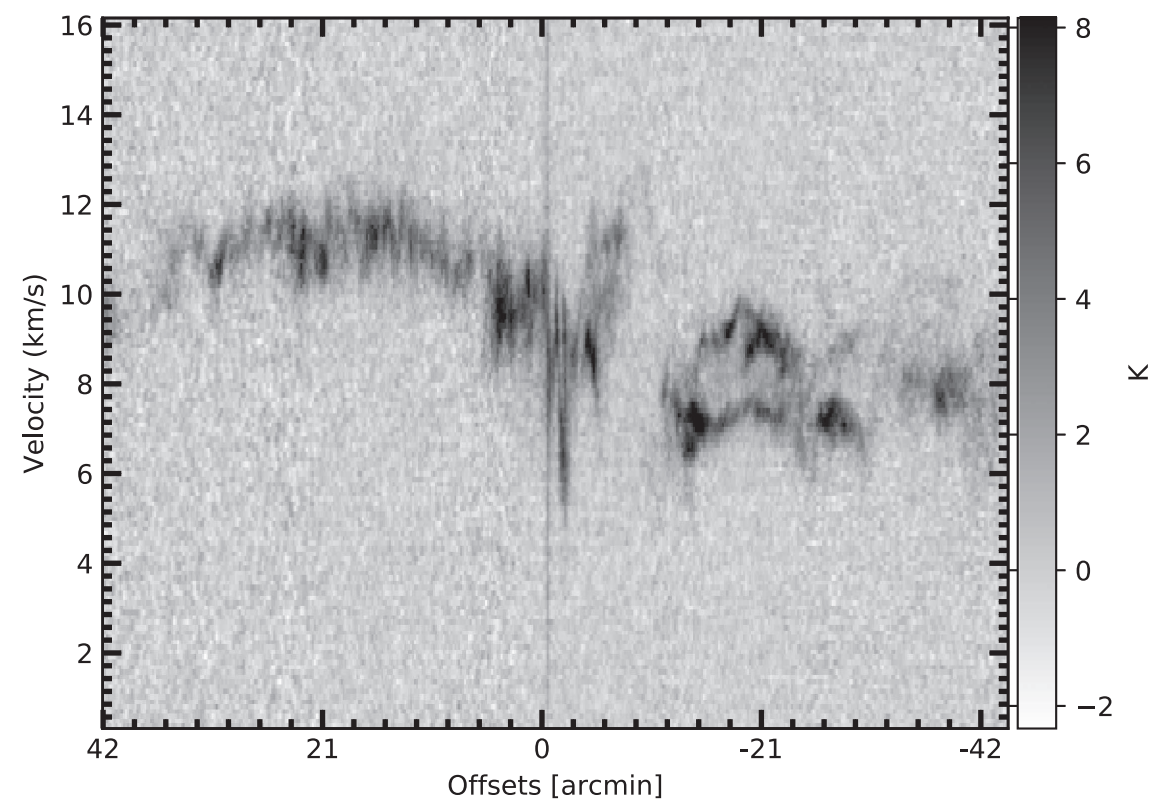

Figure 22. Same as Figure 20 but for $\mathrm{C}^{18} \mathrm{O}(1-0)$.

column densities of (1-3) $\times 10^{17} \mathrm{~cm}^{-2}$ characterize the ISF south of the ONC. The L1641 region, south of $-6^{\circ} 10^{\prime}$, shows relatively low column densities of about $10^{17} \mathrm{~cm}^{-2}$, and there are noticeable increases to $(2-3) \times 10^{17} \mathrm{~cm}^{-2}$ in the L1641-N and NGC 1999 clusters.

\subsection{The $\left.{ }^{12} \mathrm{CO}(1-0)\right)^{13} \mathrm{CO}(1-0)$ Line Ratio}

In our maps, ${ }^{12} \mathrm{CO}(1-0)$ is usually optically thick and thus more sensitive to the line width and turbulence in the observed gas. By contrast, ${ }^{13} \mathrm{CO}(1-0)$ is in general optically thin (Section 5.2) and more sensitive to column density variations (Pineda et al. 2008). The ${ }^{12} \mathrm{CO}-\mathrm{to}^{-13} \mathrm{CO}$ ratio can therefore provide indications of the physical state of the molecular gas. Figure 25 shows the variation in the ratio of ${ }^{12} \mathrm{CO}(1-0)$ integrated intensity (hereafter $W\left({ }^{12} \mathrm{CO}\right)$ ) to ${ }^{13} \mathrm{CO}(1-0)$ (hereafter $\left.W\left({ }^{13} \mathrm{CO}\right)\right)$ across the mapped region of the Orion A cloud. Pixels with a signal-to-noise ratio $<5$ in the integrated intensity maps of Figures 5 and 6 are masked (white). The same integration range $\left(2.5-15 \mathrm{~km} \mathrm{~s}^{-1}\right)$ was used for both the ${ }^{12} \mathrm{CO}$ and ${ }^{13} \mathrm{CO}$ cubes. Factors contributing less dramatically to $W\left({ }^{12} \mathrm{CO}\right)$ and $W\left({ }^{13} \mathrm{CO}\right)$ include the $\mathrm{CO}$ abundance and the gas excitation. Excitation is regulated by gas volume density so that low densities can lead to subthermal excitation, while at low temperatures, $\mathrm{CO}$ depletion can reduce the gas-phase abundance and resulting emission intensity. In relatively unshielded regions, dissociation by FUV radiation can also reduce the abundance.

In Figure 25 , values of the $W\left({ }^{12} \mathrm{CO}\right) / W\left({ }^{13} \mathrm{CO}\right)$ ratio are $<6$ across most of the cloud, including the major ISF filament. In some regions, the ratio can be as low as $\sim 2$ (e.g., OMC-3, OMC-4, L1641-N, NGC 1999). A comparison with Figures 6 and 24 shows strong ${ }^{13} \mathrm{CO}$ emission consistent with high column densities in these same regions. Overall, the trends are qualitatively similar to those noted by Pineda et al. (2008) in Perseus.

By contrast, much higher ratios $(\sim 15)$ are found in the PDR regions to the east of the ONC and OMC-4, where the photoeroded pillars are located. Other high-ratio regions include the area northwest of the "Fingers" and southeast of NGC 1999. These are probably due to the combination of radiative transfer, selective photodissociation, and chemical fractionation that is also seen in the Orion B cloud (Pety et al. 2017). A transition border $(\sim 10)$ from low ratios to high ratios can be seen surrounding the high column density gas, indicative of selective photodissociation (e.g., Shimajiri et al. 2014; Kong et al. 2015). A full understanding of gas excitation and dissociation at both large and small scales will require synthetic observations based on simulations that include radiative feedback and chemistry.

\section{Summary}

In this paper, we present the results obtained by combining new CARMA ${ }^{12} \mathrm{CO}(1-0),{ }^{13} \mathrm{CO}(1-0)$, and $\mathrm{C}^{18} \mathrm{O}(1-0)$ molecular-line observations of an extended area of the Orion A cloud with complementary NRO45 measurements of the same region. The footprint spans about $2^{\circ}$ in decl. and reaches from the OMC-3 and OMC-2 regions in the north to the NGC 1999 and L1641-C complex in the south. In particular, we provide practical details of our data combination methods and describe our checks on the effectiveness and accuracy of our processes. We find that over a carefully defined range, the observed CARMA and NRO45 fluxes can be matched using a scale factor of 1.6. Likewise, a $\Delta$-variance analysis on the product images shows no artifacts due to the data combination.

The breadth of the information on SF phenomena and processes that can be derived from the combined data sets is very obvious in the images shown. Integrated intensity maps of ${ }^{12} \mathrm{CO}(1-0)$, ${ }^{13} \mathrm{CO}(1-0)$, and $\mathrm{C}^{18} \mathrm{O}(1-0)$ emission display a range of structures typical of every stage of early stellar evolution. Channel maps and images of velocity variations across the cloud indicate an overall large-scale velocity gradient from north to south. In addition, the different structures identified in the molecular-line intensity maps often have particular kinematic signatures. The potential scope and detail for kinematic studies is illustrated by PV diagrams of the Orion ISF feature. Maps of excitation temperature and column density trace the physical properties of the Orion A cloud as a whole and of individual features by themselves and in the context of the larger cloud environment.

Perhaps most importantly, by successfully combining highresolution interferometer maps with lower-resolution singledish observations of the Orion A cloud, we have produced molecular-line images of an extended star-forming cloud with 


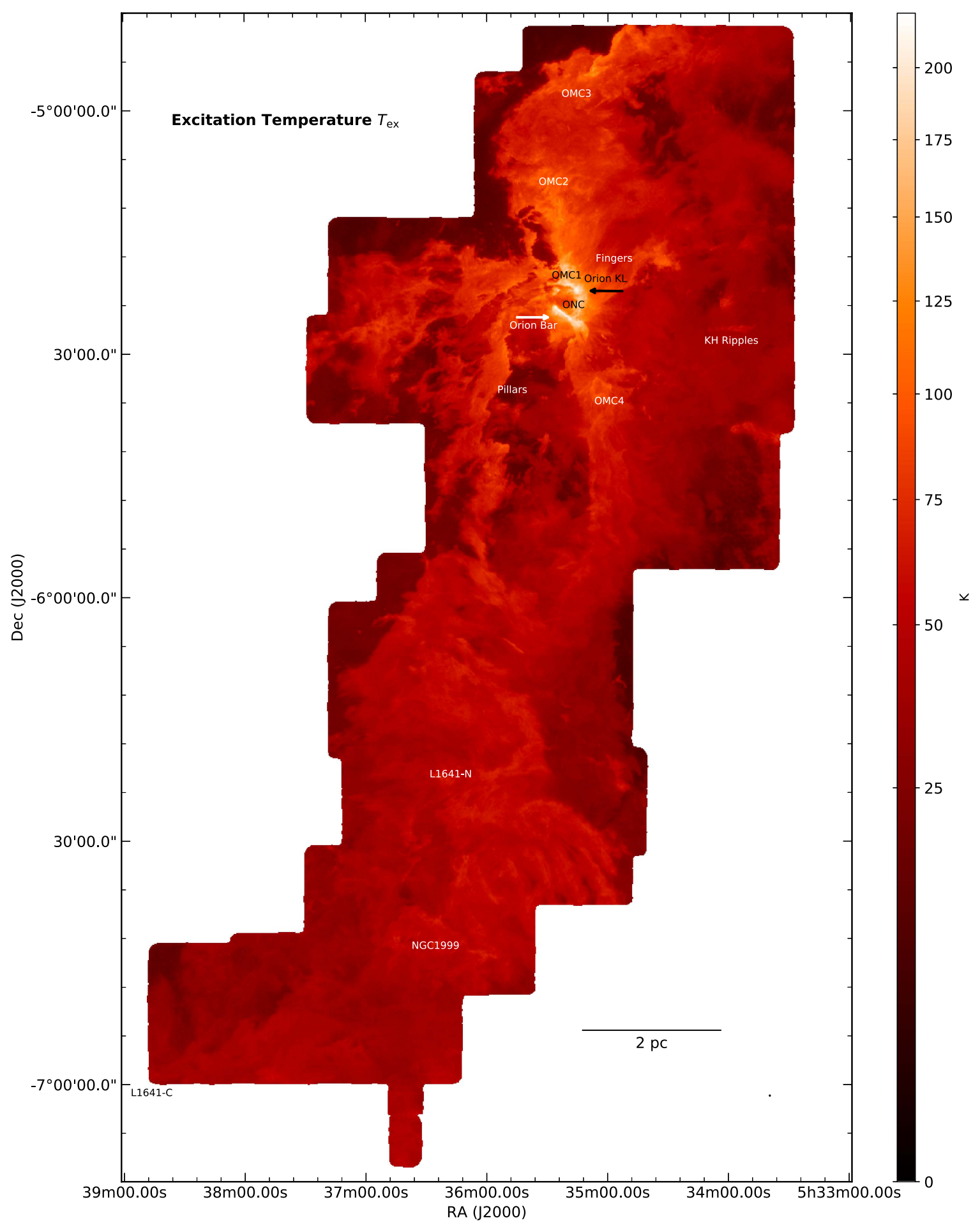

Figure 23. Excitation temperature $T_{\mathrm{ex}}$ map derived from ${ }^{12} \mathrm{CO}(1-0)$ peak intensity. The color bar is in square-root scale with units of $\mathrm{K}$.

unprecedented detail. Studies of filaments, feedback processes, and cloud-scale SF laws are already under way using these maps. There is a wealth of information to be tapped from these data. The maps presented here are currently available at https:// dataverse.harvard.edu/dataverse/CARMA-NRO-Orion, and we anticipate making all three combined Orion A data cubes publicly available within 18 months.

We thank the anonymous referee for a thorough check on the paper and helpful comments. We thank Amelia Stutz for fruitful discussions. This research was supported by the National Science Foundation, award AST-1140063, which also provided partial support for CARMA operations. CARMA operations were also supported by the California Institute of Technology, the University of California-Berkeley, the University of Illinois at Urbana-Champaign, the University of Maryland College Park, and the University of Chicago. The Nobeyama $45 \mathrm{~m}$ telescope is operated by the Nobeyama Radio Observatory, a branch of the National Astronomical Observatory of Japan. We are grateful to the staff at both NRO and 


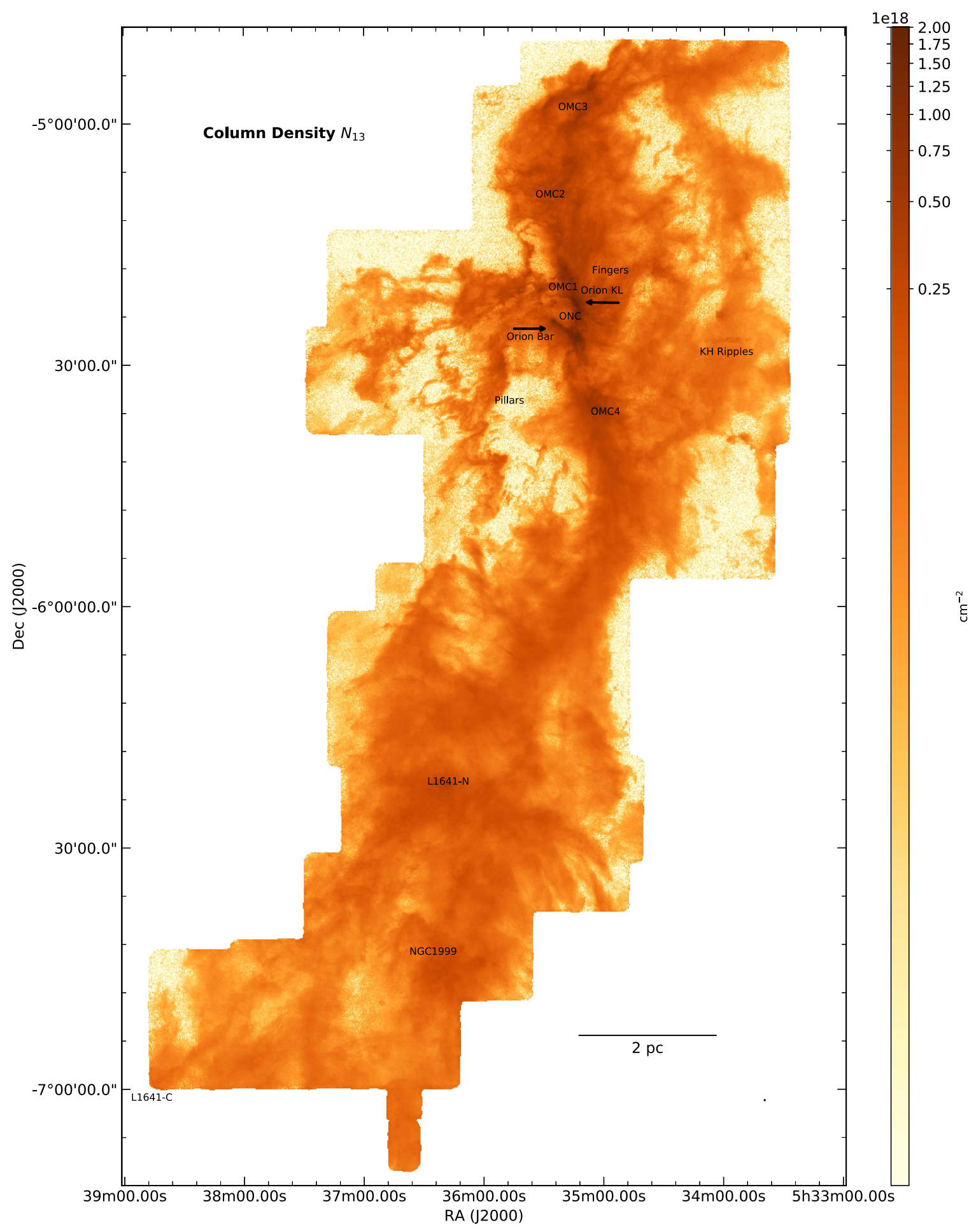

Figure 24. Map of the ${ }^{13} \mathrm{CO}$ column density, $N_{13}$, for the Orion A cloud. Units are $10^{18} \mathrm{~cm}^{-2}$.

CARMA for all their contributions. We also thank the Yale Center for Research Computing and the staff of their HPC facilities for their support. We thank Rob Gutermuth for providing the Spitzer images. We thank Alvaro Hacar for providing the ALMA data. HGA and JRF were partially funded by NSF award AST-1311825 while conducting this study. JEP acknowledges the financial support of the European Research Council (ERC; project PALs 320620). RJS acknowledges an
STFC Ernest Rutherford fellowship. VO was supported by the Deutsche Forschungsgemeinschaft (DFG) through project number 177/2-2 and central funds of the DFG priority program 1573 (ISM-SPP). STS and ASM acknowledge funding by the DFG via the Sonderforschungsbereich SFB 956 Conditions and Impact of Star Formation (subproject A4 and A6) and the Bonn-Cologne Graduate School. RSK acknowledges financial support from the DFG via SFB 881, 


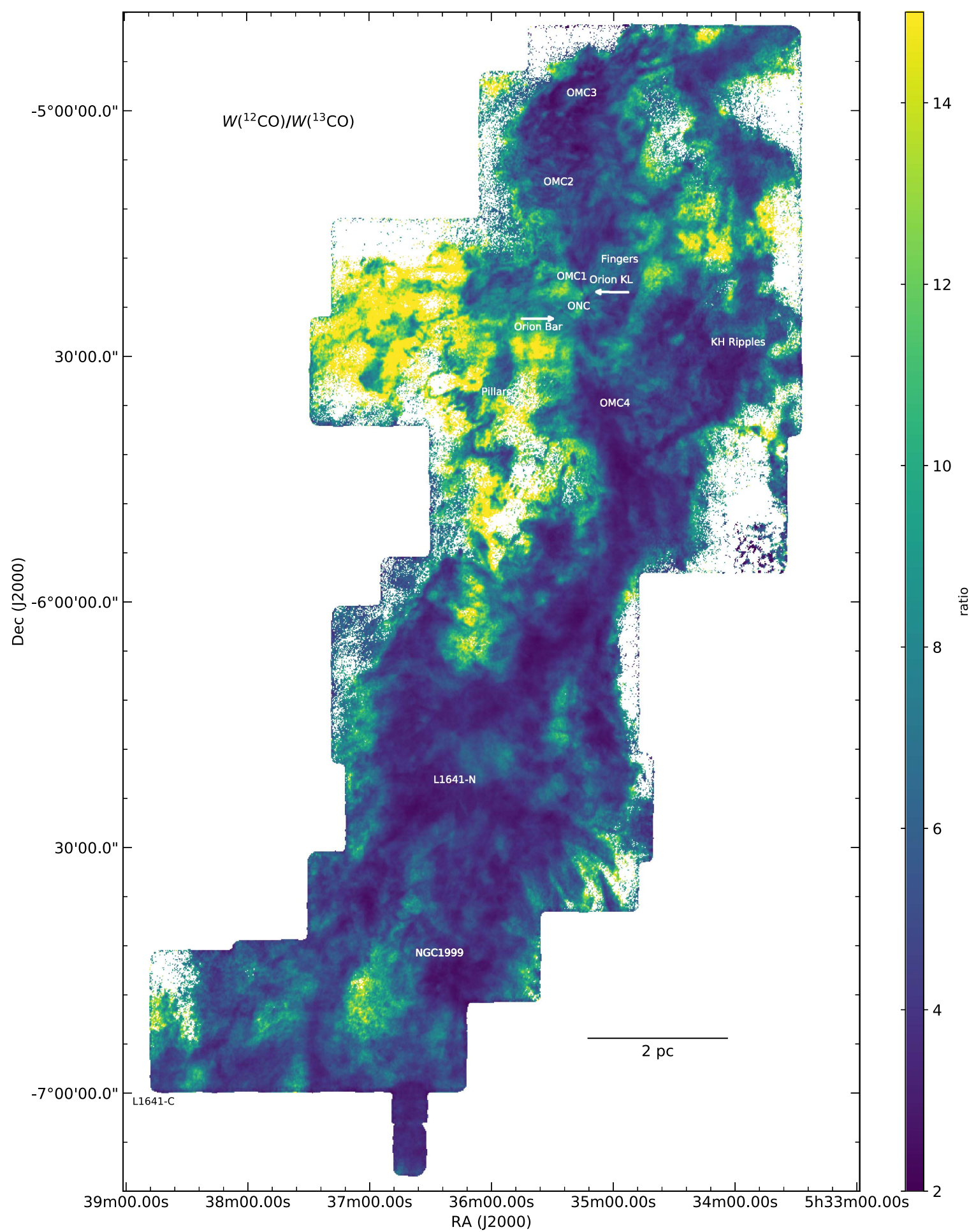

Figure 25. Map of the ratio of ${ }^{12} \mathrm{CO}(1-0)$ to ${ }^{13} \mathrm{CO}(1-0)$ integrated intensities in the Orion A cloud. Masked areas (white) indicate pixels in the maps of Figures 5 or 6 where the signal-to-noise ratio is $<5$.

"The Milky Way System" (subprojects B1, B2, and B8) and SPP 1573, "Physics of the Interstellar Medium." He is also grateful for funding from the European Research Council via the ERC Advanced Grant "STARLIGHT: Formation of the First Stars" (project number 339177). P.P. is supported by the Spanish MINECO under project AYA2017-88754-P. This research was carried out in part at the Jet Propulsion Laboratory, operated for NASA by the California Institute of Technology.

Facilities: CARMA, No:45m.
Software: Astropy (Astropy Collaboration et al. 2013), Numpy (van der Walt et al. 2011), APLpy (Robitaille \& Bressert 2012), Matplotlib (Hunter 2007).

\section{Appendix Channel Maps}

This appendix contains complete channel maps for $12 \mathrm{CO}$ (Figure 26), 13CO (Figure 27), and $\mathrm{C} 18 \mathrm{O}$ (Figure 28). These are supplementary to Figures 10-12. 


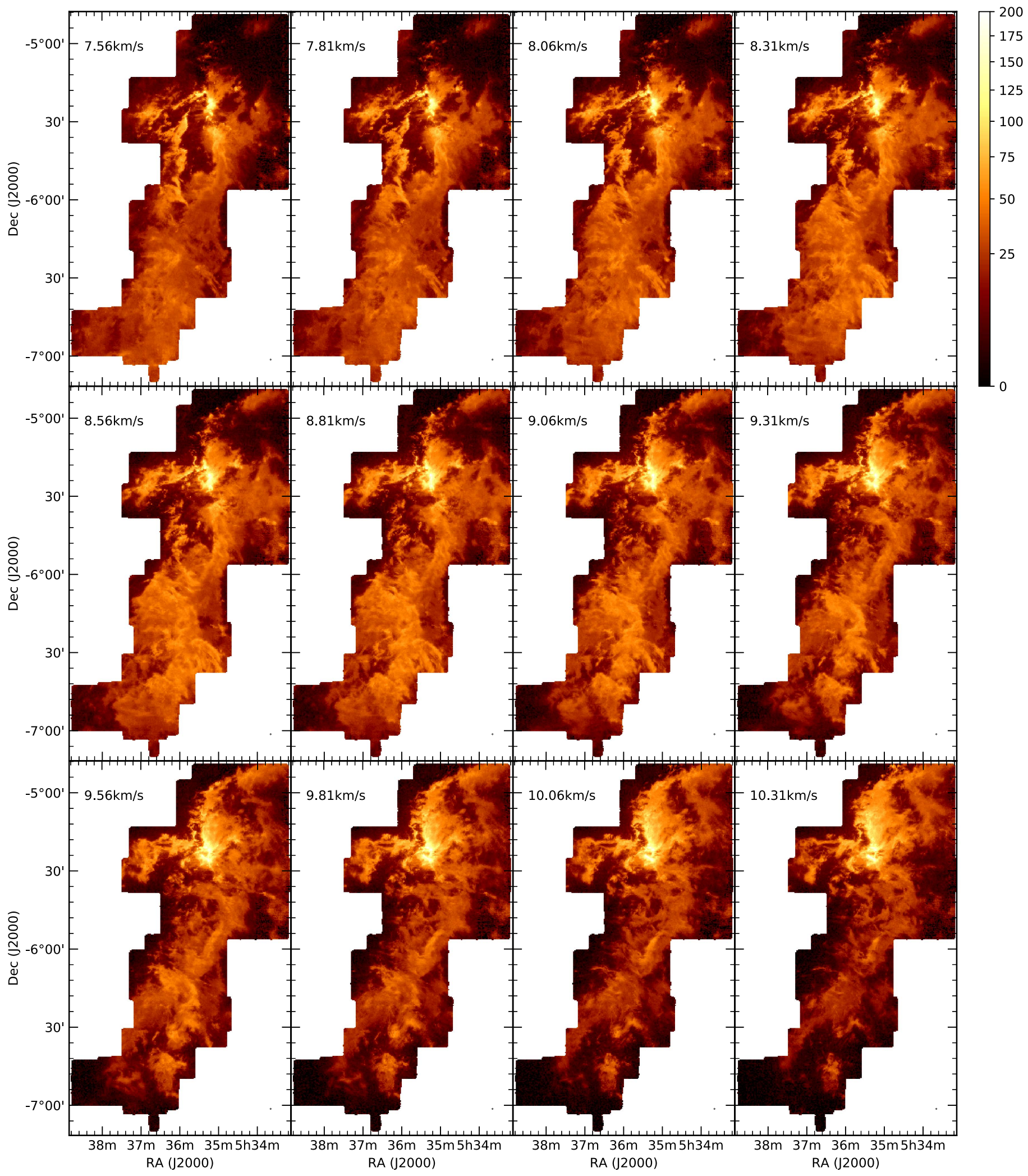

Figure 26. ${ }^{12} \mathrm{CO}(1-0)$ channel maps from $V_{\mathrm{lsr}}=7.56$ to $10.31 \mathrm{~km} \mathrm{~s}^{-1}$.

(The complete figure set (6 images) is available.) 


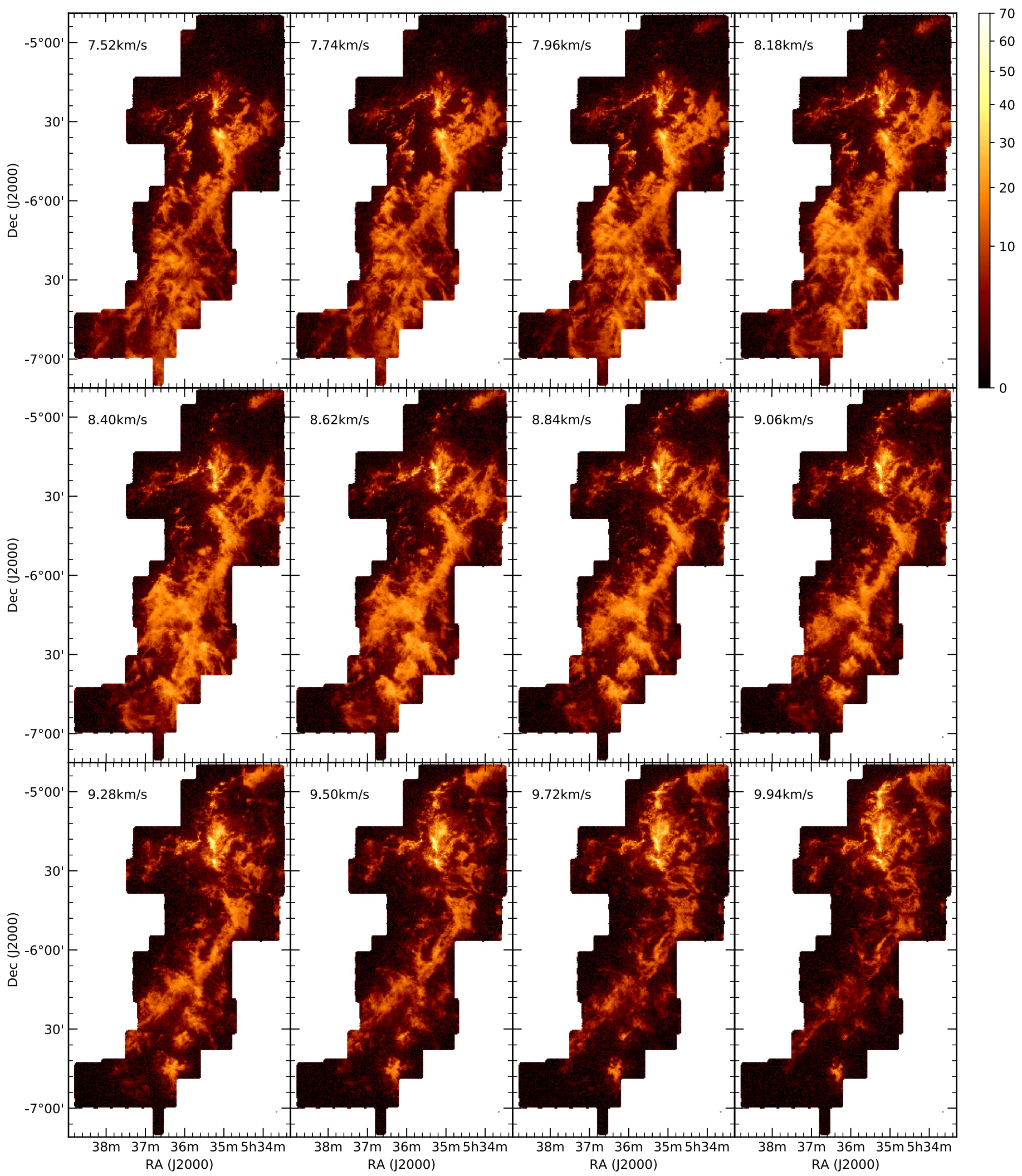

Figure 27. ${ }^{13} \mathrm{CO}(1-0)$ channel maps from $V_{\mathrm{lsr}}=7.52$ to $9.94 \mathrm{~km} \mathrm{~s}^{-1}$.

(The complete figure set (6 images) is available.) 


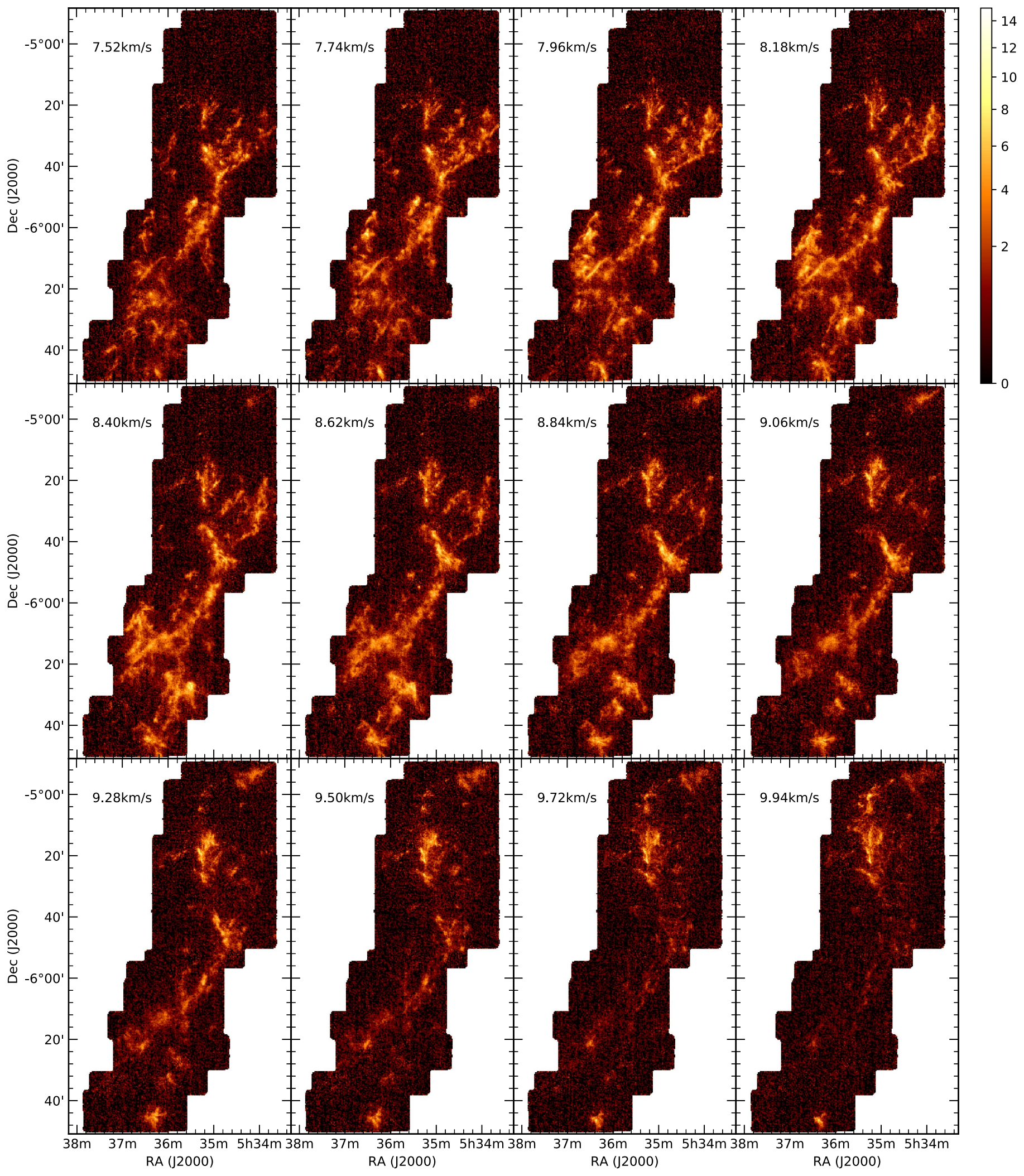

Figure 28. $\mathrm{C}^{18} \mathrm{O}(1-0)$ channel maps from $V_{\mathrm{lsr}}=7.52$ to $9.94 \mathrm{~km} \mathrm{~s}^{-1}$.

(The complete figure set (6 images) is available.) 


\section{ORCID iDs}

Héctor G. Arce (iD https://orcid.org/0000-0001-5653-7817 Jesse R. Feddersen (i) https://orcid.org/0000-0003-3810-3323 John M. Carpenter (i) https://orcid.org/0000-0003-2251-0602 Fumitaka Nakamura (iD https://orcid.org/0000-0001-5431-2294 Yoshito Shimajiri (i) https://orcid.org/0000-0001-9368-3143 Andrea Isella (i) https://orcid.org/0000-0001-8061-2207 Volker Ossenkopf-Okada (iD https://orcid.org/0000-00028351-3877

Anneila I. Sargent (iD https://orcid.org/0000-0002-4633-5098 Álvaro Sánchez-Monge (iD https://orcid.org/0000-00023078-9482

Sümeyye T. Suri (iD https://orcid.org/0000-0003-0412-8522

Jens Kauffmann (iD https://orcid.org/0000-0002-5094-6393

Jaime E. Pineda (1) https://orcid.org/0000-0002-3972-1978

Jin Koda (iD https://orcid.org/0000-0002-8762-7863

John Bally (iD https://orcid.org/0000-0001-8135-6612

Dariusz C. Lis (i) https://orcid.org/0000-0002-0500-4700

Paolo Padoan (iD https://orcid.org/0000-0002-5055-5800

Ralf Klessen (iD https://orcid.org/0000-0002-0560-3172

Steve Mairs (iD https://orcid.org/0000-0002-6956-0730

Alyssa Goodman (1) https://orcid.org/0000-0003-1312-0477

Paul Goldsmith (iD https://orcid.org/0000-0002-6622-8396

Peter Schilke (i) https://orcid.org/0000-0003-2141-5689

Peter J. Teuben (iD https://orcid.org/0000-0003-1774-3436

Adam Ginsburg (i) https://orcid.org/0000-0001-6431-9633

Rowan J. Smith (1) https://orcid.org/0000-0002-0820-1814

Jorge L. Pineda (iD https://orcid.org/0000-0001-8898-2800

Ryohei Kawabe (ib https://orcid.org/0000-0002-8049-7525

\section{References}

Allen, L. E., \& Davis, C. J. 2008, in Handbook of Star Forming Regions: The Northern Sky, Vol. 1 ed. B Reipurth (San Francisco, CA: ASP), 621

Arce, H. G., Borkin, M. A., Goodman, A. A., Pineda, J. E., \& Beaumont, C. N. 2011, ApJ, 742, 105

Arce, H. G., Borkin, M. A., Goodman, A. A., Pineda, J. E., \& Halle, M. W. 2010, ApJ, 715, 1170

Astropy Collaboration, Robitaille, T. P., Tollerud, E. J., et al. 2013, A\&A, 558, A33

Bally, J. 2008, in Handbook of Star Forming Regions: The Northern Sky, Vol. 1 ed. B. Reipurth (San Francisco, CA: ASP), 459

Bally, J., Ginsburg, A., Arce, H., et al. 2017, ApJ, 837, 60

Bally, J., Langer, W. D., Stark, A. A., \& Wilson, R. W. 1987, ApJL, 312, L45

Bensch, F., Stutzki, J., \& Ossenkopf, V. 2001, A\&A, 366, 636

Berné, O., Marcelino, N., \& Cernicharo, J. 2010, Natur, 466, 947

Berné, O., Marcelino, N., \& Cernicharo, J. 2014, ApJ, 795, 13

Bourke, T. L., Garay, G., Lehtinen, K. K., et al. 1997, ApJ, 476, 781

Buckle, J. V., Davis, C. J., di Francesco, J., et al. 2012, MNRAS, 422, 521

Burkhart, B., Falceta-Gonçalves, D., Kowal, G., \& Lazarian, A. 2009, ApJ, 693, 250

Burkhart, B., Lazarian, A., Ossenkopf, V., \& Stutzki, J. 2013, ApJ, 771, 123

Chen, X., Xiong, F., \& Yang, J. 2017, A\&A, 604, A13

Collins, D. C., Kritsuk, A. G., Padoan, P., et al. 2012, ApJ, 750, 13

Cunningham, A. J., Krumholz, M. R., McKee, C. F., \& Klein, R. I. 2018 , MNRAS, 476, 771

Da Rio, N., Tan, J. C., Covey, K. R., et al. 2016, ApJ, 818, 59

Davis, C. J., Dent, W. R. F., Matthews, H. E., Coulson, I. M., \& McCaughrean, M. J. 2000, MNRAS, 318, 952

Feddersen, J. R., et al. 2018, ApJ, submitted

Federrath, C. 2015, MNRAS, 450, 4035

Federrath, C., \& Banerjee, S. 2015, MNRAS, 448, 3297

Federrath, C., Roman-Duval, J., Klessen, R. S., Schmidt, W., \& Mac Low, M.-M. 2010, A\&A, 512, A81

Fischer, W. J., Megeath, S. T., Stutz, A. M., et al. 2013, AN, 334, 53

Ginsburg, A., Bally, J., \& Williams, J. P. 2011, MNRAS, 418, 2121

Goicoechea, J. R., Cuadrado, S., Pety, J., et al. 2017, arXiv:1707.08869
Goicoechea, J. R., Teyssier, D., Etxaluze, M., et al. 2015, ApJ, 812, 75

Hennebelle, P., \& Falgarone, E. 2012, A\&ARv, 20, 55

Heyer, M., \& Dame, T. M. 2015, ARA\&A, 53, 583

Hunter, J. D. 2007, CSE, 9, 90

Ikeda, N., Sunada, K., \& Kitamura, Y. 2007, ApJ, 665, 1194

Johnstone, D., \& Bally, J. 1999, ApJL, 510, L49

Kawamura, J., Hunter, T. R., Tong, C.-Y. E., et al. 2002, A\&A, 394, 271

Kennicutt, R. C., \& Evans, N. J. 2012, ARA\&A, 50, 531

Kirk, H., Friesen, R. K., Pineda, J. E., et al. 2017, ApJ, 846, 144

Klessen, R. S., \& Glover, S. C. O. 2016, Saas Fee Lecture Notes, 43, 85

Koda, J., Sawada, T., Wright, M. C. H., et al. 2011, ApJS, 193, 19

Kong, S., Lada, C. J., Lada, E. A., et al. 2015, ApJ, 805, 58

Kounkel, M., Hartmann, L., Loinard, L., et al. 2017, ApJ, 834, 142

Kowal, G., Lazarian, A., \& Beresnyak, A. 2007, ApJ, 658, 423

Krumholz, M. R., Bate, M. R., Arce, H. G., et al. 2014, in Protostars and

Planets VI, ed. H. Beuther et al. (Tucson, AZ: Univ. Arizona Press), 243

Krumholz, M. R., \& McKee, C. F. 2005, ApJ, 630, 250

Li, H., Li, D., Qian, L., et al. 2015, ApJS, 219, 20

Li, H.-B., Jiang, H., Fan, X., Gu, Q., \& Zhang, Y. 2017, NatAs, 1, 0158

Li, P. S., Klein, R. I., \& McKee, C. F. 2018, MNRAS, 473, 4220

Lombardi, M., Bouy, H., Alves, J., \& Lada, C. J. 2014, A\&A, 566, A45

López-Sepulcre, A., Taquet, V., Sánchez-Monge, Á., et al. 2013, A\&A, 556, A62

Loren, R. B. 1979, ApJL, 234, L207

Mac Low, M.-M., \& Klessen, R. S. 2004, RvMP, 76, 125

McKee, C. F., \& Ostriker, E. C. 2007, ARA\&A, 45, 565

McMullin, J. P., Waters, B., Schiebel, D., Young, W., \& Golap, K. 2007, in ASP Conf. Ser. 376, Astronomical Data Analysis Software and Systems XVI, ed. R. A. Shaw, F. Hill, \& D. J. Bell (San Francisco, CA: ASP), 127

Megeath, S. T., Gutermuth, R., Muzerolle, J., et al. 2012, AJ, 144, 192

Meingast, S., Alves, J., Mardones, D., et al. 2016, A\&A, 587, A153

Menten, K. M., Reid, M. J., Forbrich, J., \& Brunthaler, A. 2007, A\&A, 474,515

Minamidani, T., Nishimura, A., Miyamoto, Y., et al. 2016, Proc. SPIE, 9914, $99141 \mathrm{Z}$

Nakamura, F., \& Li, Z.-Y. 2007, ApJ, 662, 395

Nakamura, F., Miura, T., Kitamura, Y., et al. 2012, ApJ, 746, 25

Narayanan, G., Snell, R., \& Bemis, A. 2012, MNRAS, 425, 2641

O’Dell, C. R., Kollatschny, W., \& Ferland, G. J. 2017, ApJ, 837, 151

Ossenkopf, V., Krips, M., \& Stutzki, J. 2008, A\&A, 485, 917

Padoan, P., Federrath, C., Chabrier, G., et al. 2014, in Protostars and Planets VI, ed. H. Beuther et al. (Tucson, AZ: Univ. Arizona Press), 77

Padoan, P., Haugbølle, T., \& Nordlund, A. 2012, ApJL, 759, L27

Padoan, P., Haugbølle, T., Nordlund, A., \& Frimann, S. 2017, ApJ, 840, 48

Pellegrini, E. W., Baldwin, J. A., Ferland, G. J., Shaw, G., \& Heathcote, S. 2009, ApJ, 693, 285

Peng, T.-C., Wyrowski, F., Zapata, L. A., Güsten, R., \& Menten, K. M. 2012, A\&A, 538, A12

Pety, J., Guzmán, V. V., Orkisz, J. H., et al. 2017, A\&A, 599, A98

Pineda, J. E., Caselli, P., \& Goodman, A. A. 2008, ApJ, 679, 481

Pineda, J. L., Goldsmith, P. F., Chapman, N., et al. 2010, ApJ, 721, 686

Plunkett, A. L., Arce, H. G., Corder, S. A., et al. 2013, ApJ, 774, 22

Ripple, F., Heyer, M. H., Gutermuth, R., Snell, R. L., \& Brunt, C. M. 2013, MNRAS, 431, 1296

Robitaille, T., \& Bressert, E. 2012, APLpy: Astronomical Plotting Library in Python, Astrophysics Source Code Library, ascl:1208.017

Rodriguez-Franco, A., Martin-Pintado, J., \& Fuente, A. 1998, A\&A, 329, 1097

Roy, A., Martin, P. G., Polychroni, D., et al. 2013, ApJ, 763, 55

Salji, C. J., Richer, J. S., Buckle, J. V., et al. 2015, MNRAS, 449, 1782

Sault, R. J., Teuben, P. J., \& Wright, M. C. H. 1995, in ASP Conf. Ser. 77, Astronomical Data Analysis Software and Systems IV, ed. R. A. Shaw, H. E. Payne, \& J. J. E. Hayes (San Francisco, CA: ASP), 433

Schleicher, D. R. G., \& Stutz, A. 2018, MNRAS, 475, 121

Shimajiri, Y., Kawabe, R., Takakuwa, S., et al. 2011, PASJ, 63, 105

Shimajiri, Y., Kitamura, Y., Nakamura, F., et al. 2015a, ApJS, 217, 7

Shimajiri, Y., Kitamura, Y., Saito, M., et al. 2014, A\&A, 564, A68

Shimajiri, Y., Sakai, T., Kitamura, Y., et al. 2015b, ApJS, 221, 31

Shimajiri, Y., Sakai, T., Tsukagoshi, T., et al. 2013, ApJL, 774, L20

Shimajiri, Y., Takahashi, S., Takakuwa, S., Saito, M., \& Kawabe, R. 2008, ApJ, 683, 255

Shimajiri, Y., Takahashi, S., Takakuwa, S., Saito, M., \& Kawabe, R. 2009, PASJ, 61, 1055

Smith, R. J., Glover, S. C. O., Klessen, R. S., \& Fuller, G. A. 2016, MNRAS, 455, 3640

Sorai, K., Sunada, K., Okumura, S. K., et al. 2000, Proc. SPIE, 4015, 86 
Stanke, T., \& Williams, J. P. 2007, AJ, 133, 1307

Stutz, A. M., \& Gould, A. 2016, A\&A, 590, A2

Stutz, A. M., Tobin, J. J., Stanke, T., et al. 2013, ApJ, 767, 36

Stutzki, J., Bensch, F., Heithausen, A., Ossenkopf, V., \& Zielinsky, M. 1998, A\&A, 336, 697

Takahashi, S., Saito, M., Ohashi, N., et al. 2008, ApJ, 688, 344

Tang, X. D., Henkel, C., Menten, K. M., et al. 2018, A\&A, 609, A16
Thum, C., Lemke, D., Fahrbach, U., \& Frey, A. 1978, A\&A, 65, 207

Tobin, J. J., Stutz, A. M., Manoj, P., et al. 2016, ApJ, 831, 36

van der Walt, S., Colbert, S. C., \& Varoquaux, G. 2011, CSE, 13, 22

Ward-Thompson, D., Pattle, K., Bastien, P., et al. 2017, ApJ, 842, 66

Williams, J. P., Plambeck, R. L., \& Heyer, M. H. 2003, ApJ, 591, 1025

Wilson, B. A., Dame, T. M., Masheder, M. R. W., \& Thaddeus, P. 2005, A\&A, 430,523 